\title{
Mena regulates the LINC complex to control actin-nuclear lamina associations, trans-nuclear membrane signalling and cancer gene expression
}

Frederic Li Mow Chee ${ }^{1}$, Bruno Beernaert ${ }^{1}$, Alexander Loftus ${ }^{1}$, Yatendra Kumar ${ }^{2}$, Billie G. C. Griffith $^{1}$, Jimi C. Wills ${ }^{1}$, Ann P. Wheeler ${ }^{3}$, J. Douglas Armstrong ${ }^{4}$, Maddy Parsons ${ }^{5}$, Irene M. Leigh $^{6,7}$, Charlotte M. Proby ${ }^{6}$, Alex von Kriegsheim ${ }^{1}$, Wendy A. Bickmore ${ }^{2}$, Margaret C. Frame ${ }^{1, *}$ \& Adam Byron ${ }^{1 / *}$

Interactions between cells and the extracellular matrix, mediated by integrin adhesion complexes (IACs), play key roles in cancer progression and metastasis. We investigated systems-level changes in the integrin adhesome during metastatic progression of a patient-derived cutaneous squamous cell carcinoma (cSCC), and found that the actin regulatory protein Mena is enriched in IACs in metastatic CSCC cells. Mena is connected within a subnetwork of actin-binding proteins to the LINC complex component nesprin-2, with which it interacts and co-localises at the nuclear envelope of metastatic cells. Moreover, Mena potentiates the interactions of nesprin-2 with the actin cytoskeleton and the nuclear lamina. CRISPR-mediated Mena depletion causes altered nuclear morphology, reduces tyrosine phosphorylation of the nuclear membrane protein emerin and downregulates expression of the immunomodulatory gene PTX 3 via the recruitment of its enhancer to the nuclear periphery. We have uncovered an unexpected novel role for Mena at the nuclear membrane, where it controls the LINC complex, nuclear architecture, chromatin repositioning and cancer gene expression. This is the first description of an adhesion protein regulating gene transcription via direct signalling across the nuclear envelope.

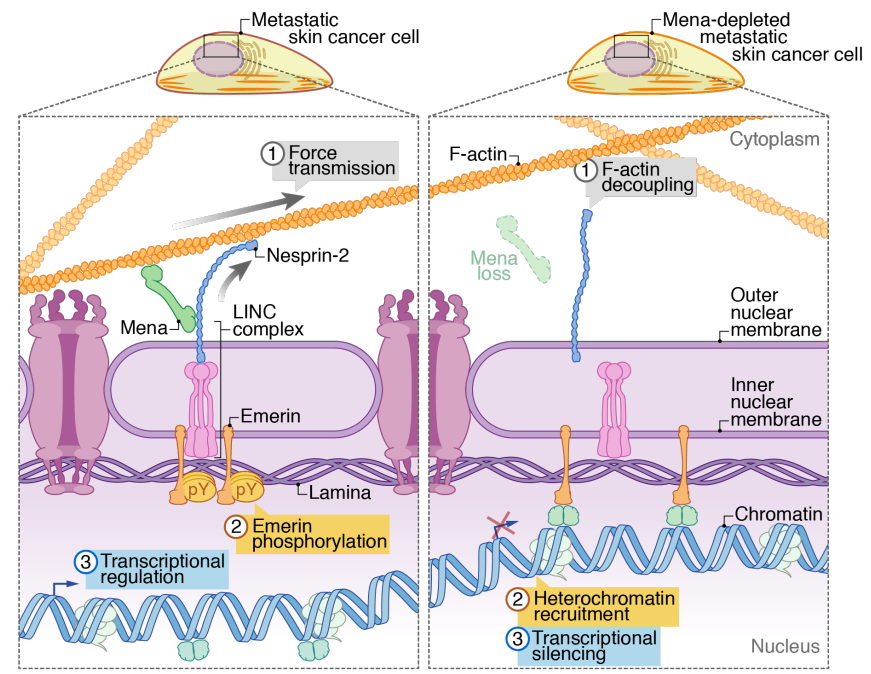

\footnotetext{
${ }^{1}$ Cancer Research UK Edinburgh Centre, Institute of Genetics and Cancer, University of Edinburgh, Edinburgh EH4 2XR, UK. ${ }^{2} \mathrm{MRC}$ Human Genetics Unit, Institute of Genetics and Cancer, University of Edinburgh, Edinburgh EH4 2XU, UK. ${ }^{3}$ Advanced Imaging Resource, Institute of Genetics and Cancer, University of Edinburgh, Edinburgh EH4 2XU, UK. ${ }^{4}$ Simons Initiative for the Developing Brain, School of Informatics, University of Edinburgh, Edinburgh EH8 9YL, UK. ${ }^{5}$ Randall Centre for Cell and Molecular Biophysics, King's College London, London SE1 1UL, UK. ${ }^{6}$ Division of Molecular and Clinical Medicine, School of Medicine, University of Dundee, Dundee DD1 4HN, UK. 'Institute of Dentistry, Barts and the London School of Medicine and Dentistry, Queen Mary University of London, London E1 2AT, UK. *email: adam.byron@ed.ac.uk or m.frame@ed.ac.uk
} 


\section{INTRODUCTION}

The execution of metastasis, a process whereby malignant cells disseminate and colonise secondary sites, requires continuous interactions between cell-surface receptors and the extracellular matrix (ECM $)^{1,2}$. CellECM interactions are mediated by transmembrane adhesion receptors, such as integrins, which associate with intracellular scaffolding, signalling and cytoskeletal proteins in adhesion complexes ${ }^{3,4}$. These multiprotein complexes function at focal adhesions to initiate and modulate adhesion signalling pathways and to control a range of cellular processes including cell migration, proliferation and differentiation ${ }^{5,6}$. Adhesion complexes are spatiotemporally regulated by biomechanical and biochemical cues in the surrounding microenvironment, acting as mechanosensory modules that physically and functionally link the ECM and the actomyosin cytoskeleton $^{4,7}$.

The set of proteins that associate with integrin adhesion complexes (IACs) is termed the integrin adhesome, which serves as a conceptual starting point for the systems-level analysis of adhesion proteins. A literature-curated adhesome database, compiled from multiple studies that used various cell types, analytical techniques and experimental settings, documents 232 adhesion-related proteins ${ }^{8,9}$. The empirical characterisation of adhesion-site composition has been enabled by the development of methods for the biochemical isolation and mass spectrometric quantification of IACs ${ }^{10-14}$ and has revealed an unanticipated molecular complexity and diversity of IAC proteomes ${ }^{15-19}$. Integration of multiple fibronectin-induced IAC proteomes in silico enabled the construction of an experimentally defined meta-adhesome database, consisting of 2,412 proteins ${ }^{20}$. A subset of the meta-adhesome, termed the consensus adhesome, contains 60 core adhesion proteins that are frequently present in IAC proteomes ${ }^{20}$.

The consensus adhesome is enriched in proteins with classical adhesion-related functions and those containing actin-binding domains; these can be divided into four interconnected axes characterised by focal adhesion kinase (FAK)-paxillin, talin-vinculin, integrin-linked kinase-kindlin and $\alpha$-actinin-zyxinvasodilator-stimulated phosphoprotein (VASP) modules, forming integrin-actin structural connections ${ }^{20,21}$. In addition to well-understood functions at focal adhesions, studies have described the localisation of certain actin-binding and actin regulatory adhesion proteins distal to IACs, such as in or around the nucleus of tumour cells. For example, $\alpha$-actinin, migfilin and zyxin, which have roles in the integrin-actin structural connection, have been reported to localise to the nucleus in tumour cells ${ }^{22-25}$, while Arp2/3 and the actin-bundling protein fascin have been described at the perinuclear region during confined cell migration ${ }^{26,27}$. There are likely multiple levels and mechanisms of dysregulation of integrin adhesomes in pathophysiological cell states, including altered expression ${ }^{28,29}$ and potentially altered subcellular localisation of adhesome components ${ }^{22,30-}$ ${ }^{34}$, and these remain to be fully defined. An outstanding question in cell biology is the function of adhesion and actin-binding adhesome components in the nucleus, or proximal to the nuclear membrane.

Mechanical stress is transmitted from the plasma membrane via the cytoskeleton to the linker of nucleoskeleton and cytoskeleton (LINC) complex at the nuclear envelope ${ }^{35}$. The LINC complex consists of the inner nuclear membrane proteins SUN1 and SUN2, the LAP2-emerin-MAN1 (LEM) domain protein emerin and the outer nuclear membrane nesprin family proteins, nesprin-1, $-2,-3$ and -4 . Nesprins anchor the cytoskeleton to the nuclear membrane either by direct interaction with the cytoskeleton or via accessory proteins $^{35,36}$. For example, nesprin-3 interacts with intermediate filaments via plectin ${ }^{37}$, while certain actinbinding proteins, such as FH1/FH2 domain-containing protein 1 (FHOD1), enhance the interaction between nesprin-2 and actin filaments ${ }^{38}$. In many cases, cells require an intact LINC complex with effective connection to the cytoskeleton to efficiently transmit mechanical stress to the nucleus to elicit nuclear mechanoresponses, such as nuclear deformation and repositioning, chromatin and gene repositioning, histone modification and nuclear protein conformational change and post-translational modifications, such as phosphorylation ${ }^{36,39,40}$. In 
many tumour cells, for example, actin and actin-associated proteins are strongly dysregulated and accumulate in or around the nucleus ${ }^{41,42}$.

In this study, we interrogated the changes in the fibronectin-induced adhesome in cancer cells using patient-derived malignant keratinocytes from cutaneous squamous cell carcinoma (cSCC). We found that the human Enabled homologue, Mena (encoded by ENAH; also known as hMena), an actin regulatory protein of the Ena/VASP family, is enriched in the adhesome of metastatic cSCC cells compared to equivalent cells from the primary tumour. Using a network analysis approach, we found that Mena clusters within an actinbinding/regulatory module that is upregulated in metastatic cSCC cells compared to primary cSCC cells from the same patient. Our proteomic analysis connected Mena to the LINC complex component nesprin-2, and we uncovered a novel role for Mena at the perinuclear region of metastatic keratinocytes. We found that Mena localises immediately adjacent to nesprin-2, and interacts with its C-terminal spectrin repeats (SRs), at the nuclear membrane. CRISPR/Cas9-mediated Mena depletion causes reduced nesprin-2 interactions with actin and lamin A/C, and alters nuclear morphology. Moreover, Mena loss results in reduced tyrosine phosphorylation of the nuclear membrane protein emerin and regulates PTX3 (which encodes pentraxin-3 (PTX3), an immunomodulatory component of the complement system) via the recruitment of its putative enhancer region to the nuclear lamina. This study elucidates a novel connection between the adhesome component Mena and the LINC complex by which Mena regulates actin-nuclear lamina interactions, nuclear architecture, chromatin organisation and cancer gene transcription. This is the first description of an adhesion protein altering gene expression by means of direct signalling 'across' the nuclear envelope.

\section{RESULTS}

\section{Characterisation of the adhesome of cSCC.}

To define changes in the adhesome during cancer progression, we biochemically isolated IACs from malignant keratinocytes derived from human CSCC at different points in the clinical progression of the disease ${ }^{43}$. Met1 cells were derived from cSCC at the primary site, whereas Met 4 cells were derived from distant metastatic cSCC within the left axillary lymph nodes from the same immunosuppressed patient ${ }^{43}$. Met 1 and Met 4 cells were seeded onto fibronectin-coated dishes to induce formation of IACs, which were stabilised using a cellpermeable crosslinker, and cell bodies were removed by detergent extraction and application of hydrodynamic force to yield IAC fractions (Fig. 1a). Western blotting confirmed enrichment of focal adhesion proteins in fibronectin-induced IACs from both primary Met1 and metastatic Met4 cSCC cells (Fig. 1b). aV integrin (a subunit of several fibronectin-binding integrin heterodimers) and the well-characterised adhesion protein focal adhesion kinase (FAK), and its fibronectin-induced tyrosine-phosphorylated active form (FAK pY397), were present in IAC fractions, whereas proteins not typically associated with integrin-based adhesions, such as GAPDH (cytoplasmic) and histone H3 (nuclear), were not detected (Fig. 1b), indicating we had achieved effective isolation of adhesion protein complexes from patient-derived cSCC cells.

We used quantitative proteomics to characterise the composition of cSCC IACs. Liquid chromatography-coupled tandem mass spectrometry (LC-MS/MS) analysis identified 191,683 peptidespectrum matches, from which 1,727 protein groups were quantified in at least two out of three biological replicate experiments (false discovery rate $(\mathrm{FDR})<1 \%$ ) (Supplementary Table 1). Replicate experiments were well correlated (Pearson correlation coefficient $\geq 0.966, P<1 \times 10^{-307}$; Supplementary Fig. 1a), and the number of proteins identified was of a similar magnitude to IAC proteomes reported for human cell lines previously $y^{16,44}$. Integrin signalling was the most over-represented functional pathway associated with the cSCC IAC subproteome (Supplementary Fig. 1b), and of the 1,727 identified cSCC IAC proteins, 1,024 proteins were members of the meta-adhesome ${ }^{20}$ and 66 were described in the literature-curated adhesome ${ }^{9}$ (Fig. 1c). We extracted the intersection set of 56 cSCC IAC proteins reported in both the meta-adhesome and the literature- 
bioRxiv preprint doi: https://doi.org/10.1101/2021.08.31.458340; this version posted August 31, 2021. The copyright holder for this preprint (which was not certified by peer review) is the author/funder, who has granted bioRxiv a license to display the preprint in perpetuity. It is made available under aCC-BY-NC-ND 4.0 International license.

a cSCC adhesome analysis

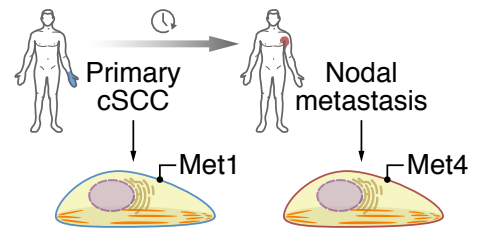

IAC induction and stabilisation

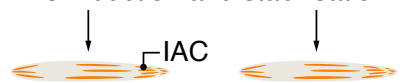

Cell body extraction and IAC isolation

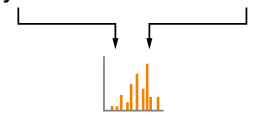

Quantitative MS analysis

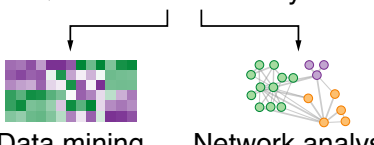

Data mining Network analysis

d Core adhesome functions

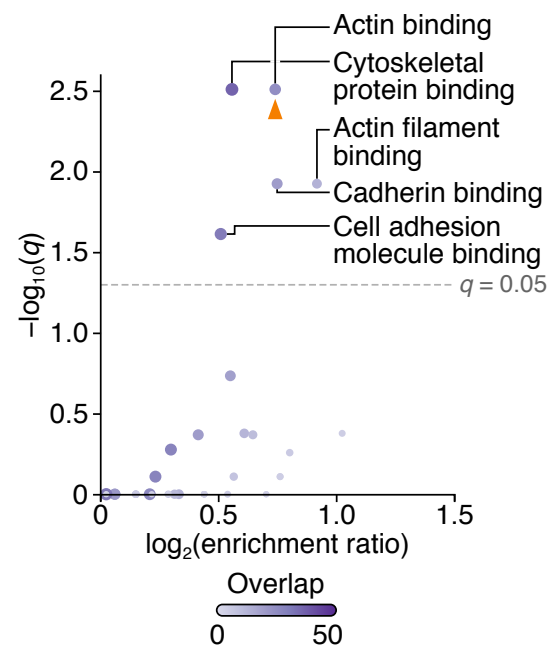

e Core adhesome clustering
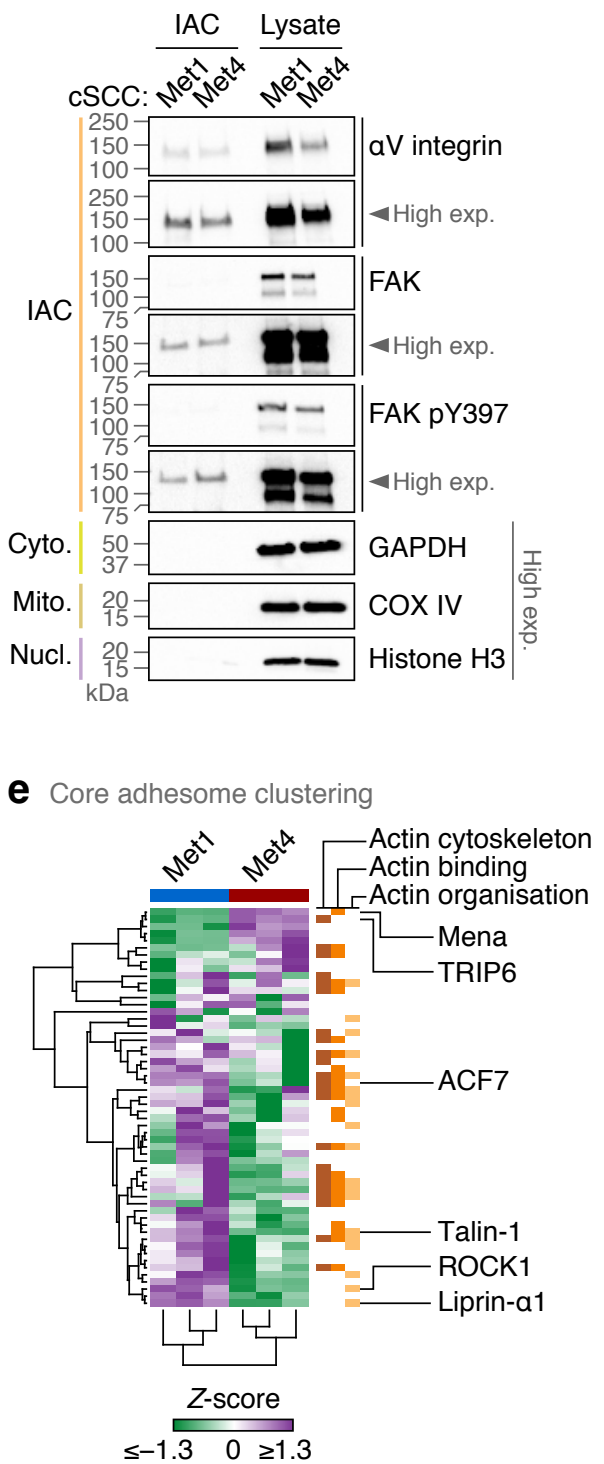

C Adhesome proteomics

f IAC regulation

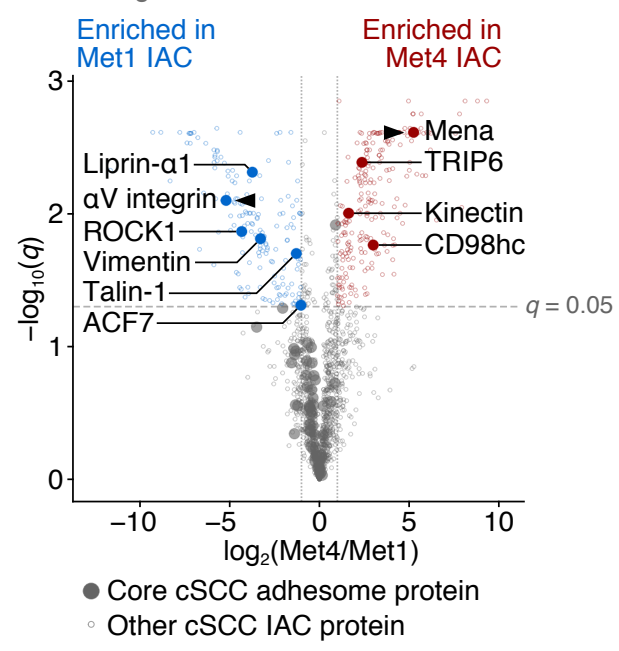

h Actin regulation cluster

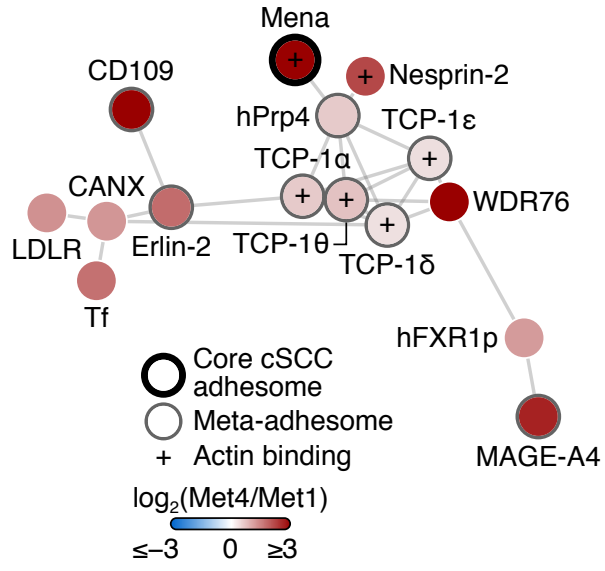

Meta-adhesome adhesome

Intersection

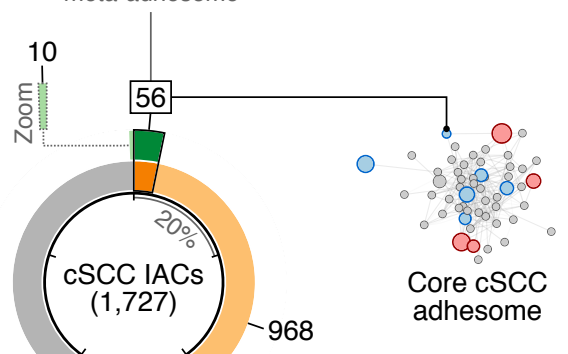

IAC subproteome
Literature-curated Curated
meta-adhesom

Other CSCC IAC protein

$\leq-30 \geq 3$ g IAC active module
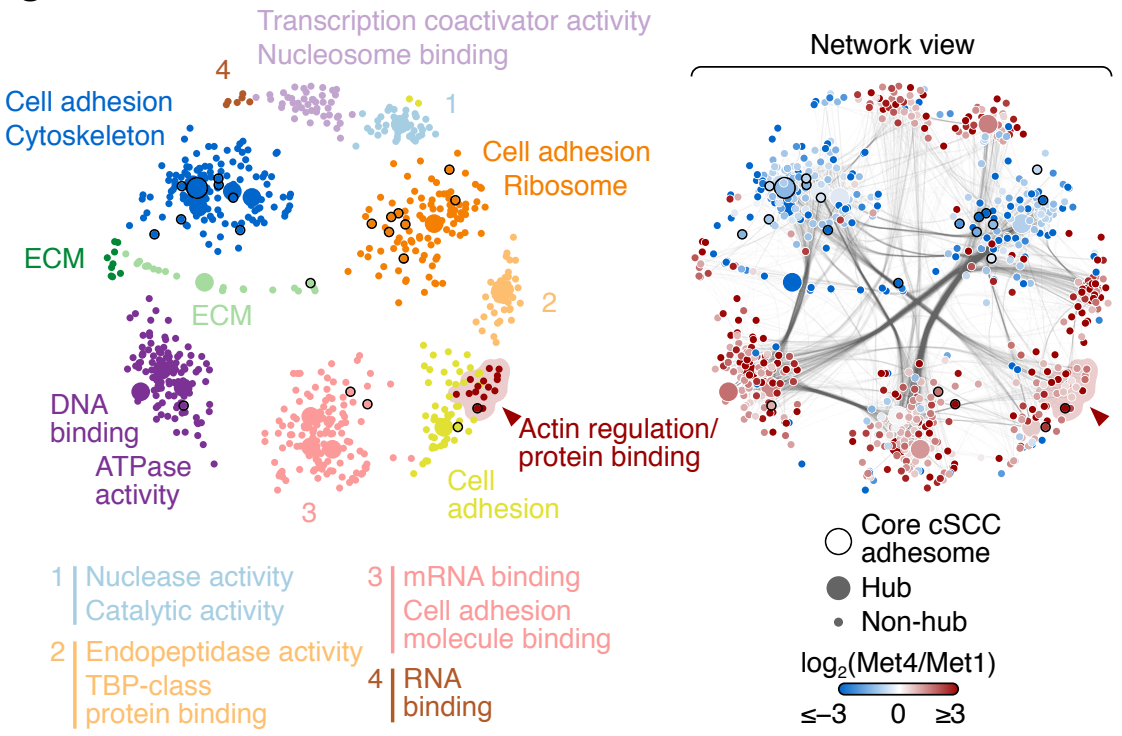

Figure 1. See next page for caption. 
Figure 1. Characterisation of a patient-derived CSCC adhesome. (a) Workflow for isolation and proteomic analysis of human cSCC IACs. (b) Enrichment of focal adhesion proteins $\alpha \mathrm{V}$ integrin, FAK and phosphorylated FAK pY397 in cSCC IACs compared to non-adhesion proteins glyceraldehyde-3-phosphate dehydrogenase (GAPDH) (cyto., cytoplasmic), cytochrome c oxidase subunit 4 (COX IV) (mito., mitochondrial) and histone H3 (nucl., nuclear) as determined by western blotting. High exp., high exposure of blot. (c) Proportion of cSCC IAC proteins (1,727 proteins identified by proteomics) annotated in the meta-adhesome (orange segments) or the literature-curated adhesome (green segments). IAC proteins in both the meta-adhesome and the literature-curated adhesome (intersection set; 56 proteins; dark segments) represent a core cSCC adhesome (see Supplementary Fig. 1c for corresponding network). Inset segment (dotted box) shows zoom of literature-curated adhesome-only segment for clarity. Tick marks indicate $20 \%$ increments. (d) Gene Ontology over-representation analysis of molecular functions in the core cSCC adhesome. Functional categories with enrichment ratio $\geq 1$ are displayed. Categories significantly enriched compared to the curated meta-adhesome reference set are labelled ( $q<0.05$, hypergeometric test with Benjamini-Hochberg correction). To reduce redundancy, the representative functional category determined using affinity propagation is indicated with an orange arrowhead. Purple shading intensity indicates size of core adhesome overlap with respective gene sets. (e) Hierarchical cluster analysis of the core cSCC adhesome. Proteins associated with actin components (actin cytoskeleton), functions (actin binding) or processes (actin organisation) are indicated (orange bars). Actin-associated proteins significantly differentially regulated between Met 1 and Met4 IACs are labelled $(q<0.05$, two-sided $t$-test with Benjamini-Hochberg correction; $n=3$ independent biological replicates). ROCK1, Rho-associated protein kinase 1. (f) Volcano plot of cSCC IAC proteins. Core adhesome proteins are indicated with large filled circles. Core adhesome proteins significantly differentially regulated between Met 1 and Met4 IACs and enriched by at least two-fold are labelled. The most enriched proteins in Met1 and Met4 IACs, respectively, are indicated with black arrowheads. CD98hc, cluster of differentiation 98 heavy chain (also known as $4 \mathrm{~F} 2$ cell-surface antigen heavy chain). (g) Maximal-scoring active module of the cSCC IAC interactome. The network was weighted by Spearman rank correlation and partitioned using the Louvain modularity maximisation method. Proteins (nodes) are coloured according to assigned cluster (left panel). Molecular functions enriched in protein clusters were determined by Gene Ontology over-representation analysis (the two most significant functional categories are labelled). For protein clusters with fewer than two significant functional categories, clusters were annotated manually with a single representative term. Network view (right panel) shows corresponding protein interactions (edge densities) in the partitioned network; nodes are coloured according to protein enrichment in Met1 or Met4 IACs. The actin regulation cluster detailed in $\mathbf{h}$ is indicated with a red arrowhead. Large nodes indicate kinless and connector hubs; black node borders indicate core adhesome proteins. (h) Subnetwork analysis of the actin regulation cluster identified by active module partitioning in $\mathbf{g}$. The weighted subnetwork was clustered using a force-directed algorithm. Nodes are coloured according to protein enrichment in Met1 or Met4 IACs. 
curated adhesome, reasoning that these adhesion proteins, which have been determined by various experimental methods from multiple cell lines ${ }^{9}$ in addition to proteomic analysis of isolated IACs, constitute a robust core subset of the cSCC adhesome (Supplementary Fig. 1c, Supplementary Table 2). These core cSCC adhesion proteins were significantly enriched for proteins associated with actin binding when compared to all proteins reported in both the meta-adhesome and the literature-curated adhesome (Fig. 1d).

Since actin-binding proteins are involved in cancer progression ${ }^{41}$, we further examined actinassociated proteins that were differentially regulated between primary and metastatic CSCC IACs. IACs from metastatic Met 4 cells were quantitatively distinct from those from primary Met 1 cells derived from the same patient (Supplementary Fig. 1a, d). Of the 56 core cSCC adhesion proteins, the actin-binding proteins talin-1 and microtubule-actin crosslinking factor 1 (also known as ACF7) were significantly depleted in metastatic Met4 IACs compared to primary Met1 IACs, whereas the actin regulatory proteins Mena and transcriptional coactivator TRIP6 were significantly enriched in Met4 IACs (Fig. 1e, f, Supplementary Fig. 1e, Supplementary Table 2). Indeed, Mena was the most enriched core adhesome protein in Met4 IACs, and the high abundance of Mena in Met4 IAC fractions was confirmed by western blotting (Supplementary Fig. 1f).

Mena has been linked with tumour progression, invasion and metastasis ${ }^{45-48}$. We therefore investigated Mena and its molecular connections in Met4 cells, using graph-based analysis to assess the topology of the cSCC adhesome network. We analysed network modularity to identify cSCC IAC proteins that may interact with each other to form modules, which have been postulated to have functional roles in particular disease phenotypes ${ }^{49}$. An active module is a well-connected subnetwork of a biological network system enriched in nodes (i.e. proteins) that change in abundance between different conditions. Hence, we reasoned that interrogating Mena in the context of an active module of the cSCC adhesome may reveal novel functions in cSCC cells.

We constructed a protein interaction network of the cSCC adhesome using curated physical proteinprotein interactions from the Biological General Repository for Interaction Datasets (BioGRID) database. A maximal-scoring subnetwork was extracted from this interaction network using a diffusion-flow emulation model implemented in the BioNet R package ${ }^{50}$. This identified the largest active module of the cSCC adhesome, which contained highly connected regions of the interaction network that show differential protein enrichment between Met1 and Met4 IACs (Fig. 1g). We next analysed the topology of the active module to infer putative functional clusters in the cSCC adhesome. The majority of clusters (7/12 identified by Louvain modularity maximisation) were annotated with cell adhesion- or cytoskeleton-related functions, with the remainder annotated with non-adhesion functions that have been linked to IAC proteins, such as transcriptional coactivator activity ${ }^{51}$ and RNA binding ${ }^{52}$ (Fig. 1g). By two independent network clustering methods, we found that Mena clustered with meta-adhesome proteins involved in filamentous actin (F-actin) binding and regulation, such as members of the chaperonin-containing TCP1 complex (CCT/TRiC) ${ }^{53}$ (Fig. 1g, h, Supplementary Fig. 1g, h). In addition, Mena was proximal in the subnetwork to nesprin-2 (encoded by SYNE2), an F-actin-binding protein of the LINC complex (Fig. 1h, Supplementary Fig. 1h). Although nesprin2 is not included in the current meta-adhesome database, other members of the LINC complex - nesprin-1, nesprin-3, SUN1 and SUN2 - are components of the meta-adhesome ${ }^{20}$. Mena and nesprin-2 thus formed part of a functional cluster that was enriched in metastatic Met4 IACs, which led us to hypothesise that Mena and nesprin-2 may operate together in cSCC cells.

\section{Mena and nesprin-2 form a complex and co-localise at the nuclear membrane.}

To test the functional relevance of the network co-clustering of Mena and nesprin-2, we first examined their localisation by immunofluorescence imaging of Met 4 cells. Spinning-disk confocal images of cells stained with anti-Mena and anti-nesprin-2 antibodies showed that, while Mena was found at a number of subcellular 
locales, including focal adhesions (Fig. 2a, orange arrowheads), it specifically co-localised with nesprin-2 at the nuclear membrane (Fig. 2a, magenta arrowheads). Orthogonal reconstruction of images acquired by superresolution structured-illumination microscopy (SIM) revealed that Mena co-localised at the apical-most region of nesprin-2 staining, where actin filaments also co-localise (Fig. 2b). Moreover, Mena coimmunoprecipitated with nesprin-2 in Met4 lysates (Fig. 2c). We observed multiple reactive bands to nesprin2 by western blotting (Fig. 2c), which we inferred are likely different nesprin-2 isoforms that have been described previously $y^{54}$. Our data indicate that Mena forms a complex with nesprin-2 and that this is likely at the nuclear membrane.

It has been shown previously that SRs (spectrin repeats) of nesprin-2 are binding sites for actinbinding proteins, namely fascin ${ }^{26}$, FHOD1 (ref. 38) and Myc box-dependent-interacting protein 1 (also known as amphiphysin 2 or bridging integrator 1$)^{55}$. SRs located at the C-terminal domain of nesprin-2 are the most likely sites of conserved protein-protein interaction ${ }^{56,57}$. We therefore tested whether Mena interacts with nesprin-2 at the C-terminal SRs by transiently transfecting Met4 cells with a GFP-tagged truncated nesprin-2 expressing only the eight C-terminal SRs 49-56 (GFP-nesprin-2G(SR49-56); based on the nesprin-2 giant isoform, nesprin-2G) (schematic representation in Fig. 2d). Isolation of associated protein complexes using GFP-Trap showed that Mena was highly enriched in GFP-nesprin-2G(SR49-56) pulldowns (Fig. 2e), indicating that Mena forms a complex with nesprin-2 C-terminal SRs. To further define the Mena binding site on nesprin-2, Met 4 cells were transiently transfected with GFP-nesprin-2G $\Delta$ SR3-50, which contains SRs 5156 of nesprin-2 (Fig. 2d), and GFP-bound protein complexes were isolated. Mena was highly enriched in GFPnesprin-2G $\Delta$ SR3-50 pulldowns (Fig. 2f), implying that Mena interacts with the region of nesprin-2 containing SRs 51-56. Taken together, we conclude that Mena binds to nesprin-2 via its C-terminal SRs, and microscopy implies this occurs at the nuclear membrane where the two proteins are juxtaposed and co-localise.

\section{Mena depletion causes LINC complex dissociation from actin and lamin A/C.}

Nesprin-2 interacts with actin, thereby coupling the actin cytoskeleton to the nuclear lamina via the LINC complex $^{35}$. The LINC complex also mediates a mechanosensitive response upon physical strain at the nuclear periphery, whereby lamin A/C is recruited to the LINC complex ${ }^{40}$. Therefore, to test the functional relevance of the interaction between Mena and the nesprin-2 component of the LINC complex, we examined whether the roles of nesprin-2 in binding the actin cytoskeleton and the nuclear lamina were regulated by Mena. We generated Mena-knockout Met4 cells (Met4 $\mathrm{Mena}^{--}$) using CRISPR/Cas9 and re-expressed the human Mena isoform variant 11a (Mena11a) in Met4 $\mathrm{Mena}^{-/-}$cells (Supplementary Fig. 2a). The Mena1la variant has an extra 63-nucleotide exon between exons 11 and 12, conferring an additional 21 amino acids to the Mena EVH2 domain ${ }^{58}$. Mena1 la was re-expressed because the main endogenous Mena-reactive band of Met 4 cells migrates similarly with exogenous Mena11a (Supplementary Fig. 2a), suggesting that Met4 cells express the Mena11a variant. We verified this by sequence analysis of cDNA corresponding to the $\mathrm{C}$-terminus of endogenous Mena obtained by reverse transcription of mRNA isolated from Met 4 cells. We observed the presence of the additional 63 nucleotides that match the Menal la variant (Supplementary Fig. 2b), thereby confirming the presence of endogenous Mena1la in Met4 cells, although expression of other Mena isoforms in Met4 cells is not excluded.

Co-immunoprecipitation of endogenous nesprin-2 revealed that depletion of Mena by CRISPR/Cas9mediated knockout inhibited the interaction of nesprin-2 with actin (Fig. 3a) and suppressed the interaction between nesprin-2 and lamin A/C (Fig. 3b). These interactions were restored by re-expression of the Mena11a variant (Fig. 3a, b); the interaction between nesprin-2 and actin was increased by the overexpression of Mena11a, implying that the level of this biochemical complex may be dictated by the amount of Mena. These 
bioRxiv preprint doi: https://doi org/10.1101/2021.08 31.458340; this version posted August 31, 2021. The copyright holder for this preprint (which was not certified by peer review) is the author/funder, who has granted bioRxiv a license to display the preprint in perpetuity. It is made available under aCC-BY-NC-ND 4.0 International license.

a Met4 confocal imaging

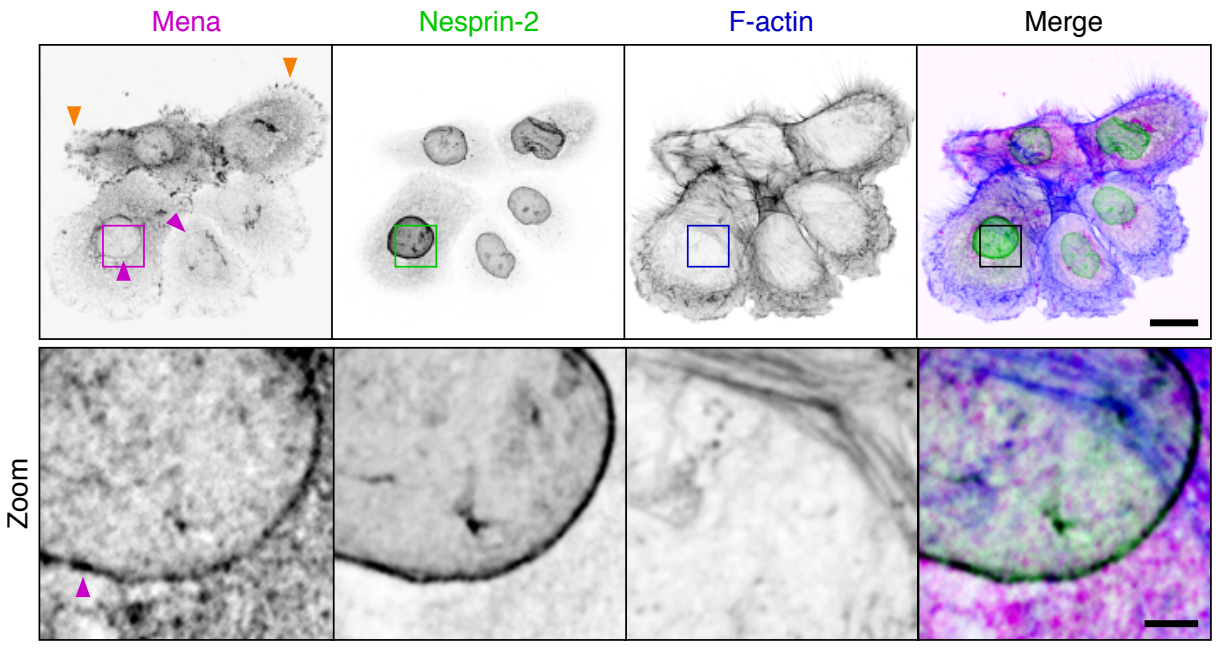

b Met4 super-resolution imaging

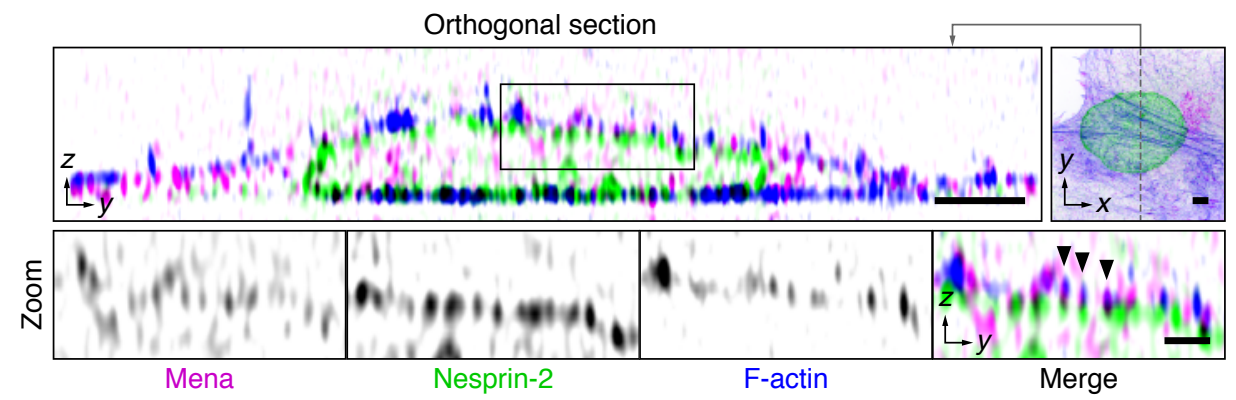

C Met4 nesprin-2 IP

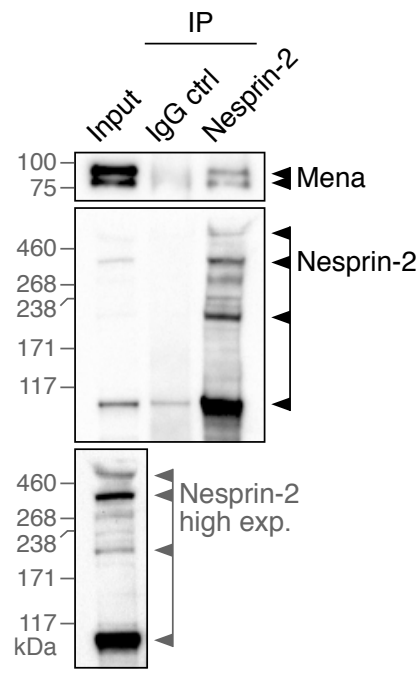

d Nesprin-2 constructs

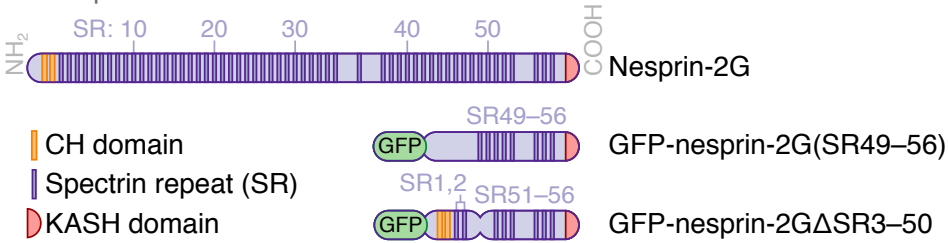

e Nesprin-2 SR49-56 pulldown

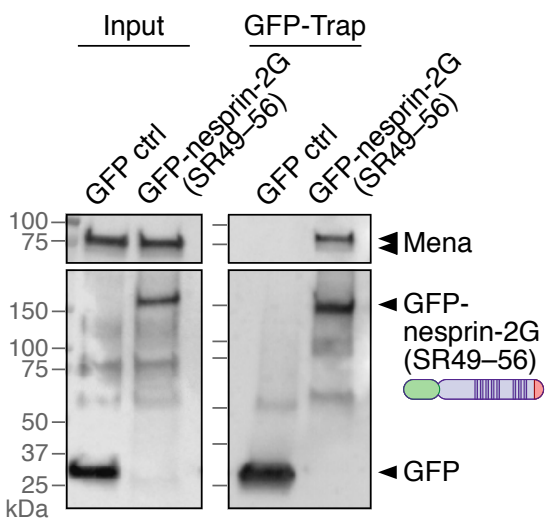

f Nesprin-2 SR51-56 pulldown Input $\frac{\text { GFP-Trap }}{2^{G}}$

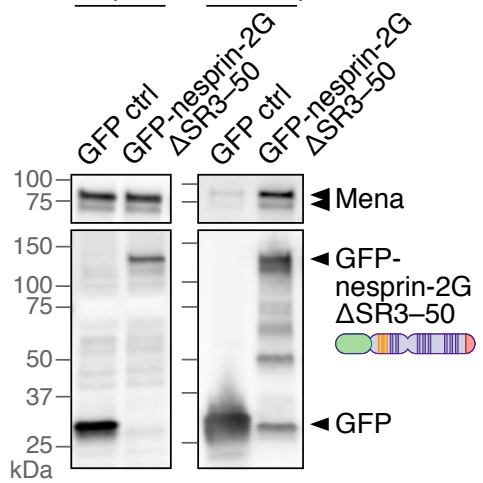

Figure 2. See next page for caption. 
bioRxiv preprint doi: https://doi.org/10.1101/2021.08.31.458340; this version posted August 31, 2021. The copyright holder for this preprint (which was not certified by peer review) is the author/funder, who has granted bioRxiv a license to display the preprint in perpetuity. It is made available under aCC-BY-NC-ND 4.0 International license.

Figure 2. Mena co-localises with nesprin-2 at the nuclear membrane and forms a biochemical complex. (a) Spinning-disk confocal imaging of Met 4 cSCC cells. Orange arrowheads indicate Mena staining at focal adhesions; magenta arrowheads indicate Mena localisation at the nuclear membrane. F-actin was detected using phalloidin. Inverted lookup tables were applied; in merged images, co-localisation of Mena (magenta) and nesprin-2 (green) is represented by black regions. Images are representative of three independent experiments. Scale bar, $20 \mu \mathrm{m}$; zoom (inset) scale bar, $3 \mu \mathrm{m}$. (b) SIM super-resolution imaging of Met 4 cells. Position of orthogonal section $(y z)$ shown in top-right image ( $x y$; grey dashed line). Black arrowheads indicate co-localisation of Mena, nesprin-2 and F-actin at the nuclear membrane. F-actin was detected using phalloidin. Inverted lookup tables were applied. Scale bar, $3 \mu \mathrm{m}$; zoom (inset) scale bar, $1 \mu \mathrm{m}$.

(c) Immunoprecipitation (IP) analysis of nesprin-2 protein complexes in Met4 cells. Anti-Mena- and anti-nesprin-2reactive species detected by western blotting are indicated with arrowheads. High exposure (high exp.) of nesprin-2 blot of Met4 lysate (input) is also shown. Western blots are representative of three independent experiments. Ctrl, control. (d) Representation of nesprin-2 domains and the nesprin-2 constructs used for pulldown experiments. Constructs are based on the nesprin-2 giant isoform (nesprin-2G). GFP-nesprin-2G(SR49-56) contains C-terminal spectrin repeats (SR)49-56; GFP-nesprin-2G $\Delta$ SR3-50 (also known as GFP-mini-nesprin-2G SR51-56) contains C-terminal SR51-56. $\mathrm{CH}$, calponin homology; KASH, Klarsicht-ANC-1-Syne homology. (e, f) Pulldown analyses of protein complexes associated with exogenous GFP-tagged nesprin-2G(SR49-56) (e) and nesprin-2G $\Delta$ SR3-50 (f) in Met4 cells. Anti-Menareactive species were enriched in nesprin-2G(SR49-56) and nesprin-2G $\Delta$ SR3-50 GFP-Trap pulldown samples but not in GFP control (Ctrl) samples. Western blots are representative of three independent experiments. 
bioRxiv preprint doi: https://doi.org/10.1101/2021.08.31.458340; this version posted August 31, 2021. The copyright holder for this preprint (which was not certified by peer review) is the author/funder, who has granted bioRxiv a license to display the preprint in perpetuity. It is made available under aCC-BY-NC-ND 4.0 International license.

a Mena-dependent nesprin-2 IP: actin

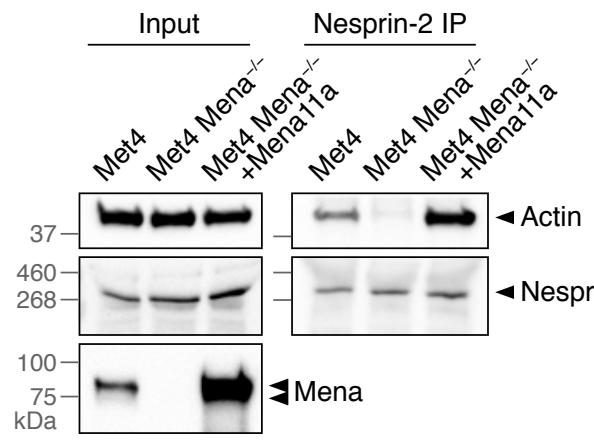

b Mena-dependent nesprin-2 IP: Iamin A/C

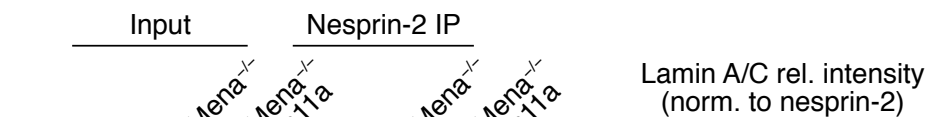

(norm. to nesprin-2)
Actin rel. intensity
(norm. to nesprin-2)

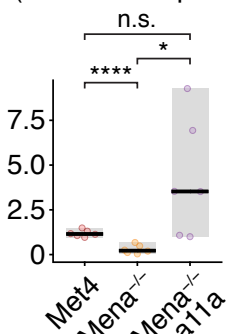

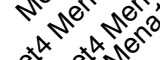

$3 e^{x}+e^{x} x$
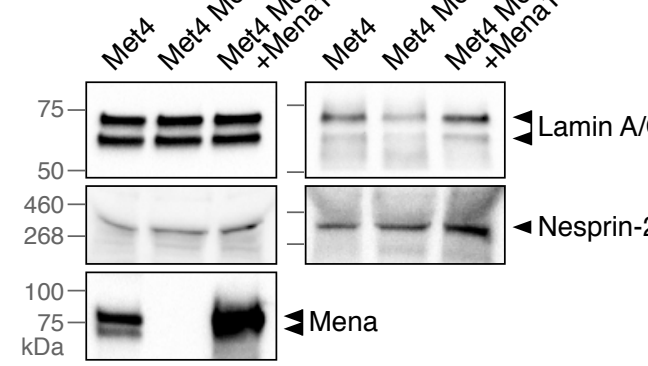

C Nuclear morphology: 3D matrix

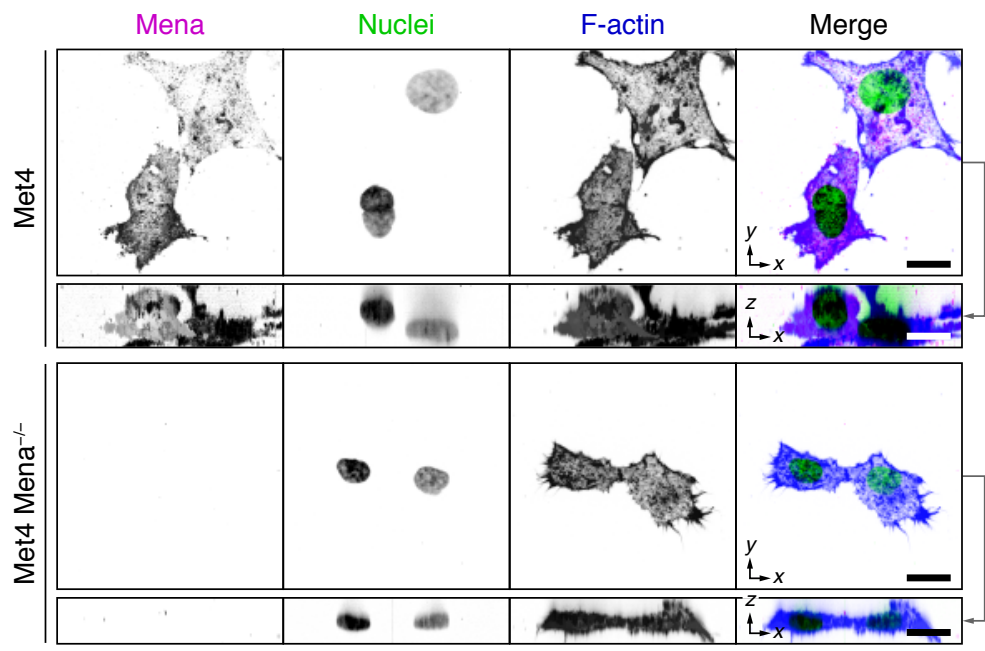

Orthogonal projection

e Nuclear morphology: 2D matrix

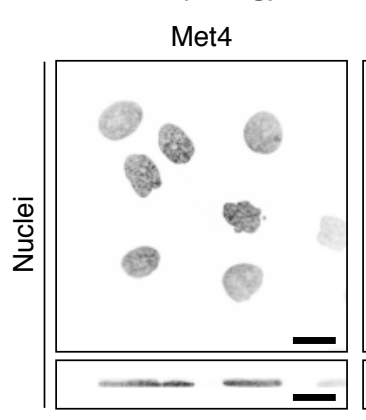

Met4 Mena-/-

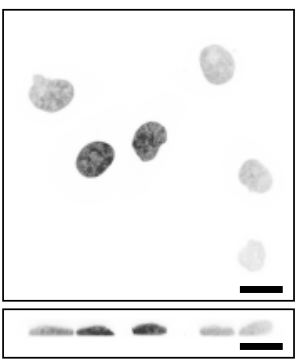

Met4 Mena ${ }^{-/-}$

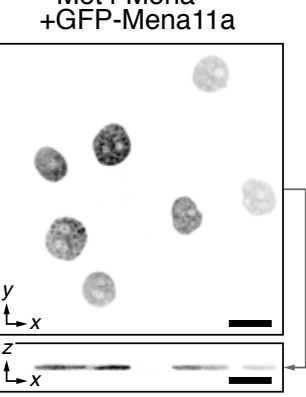

Orthogonal Orthogonal

d Nuclear volume

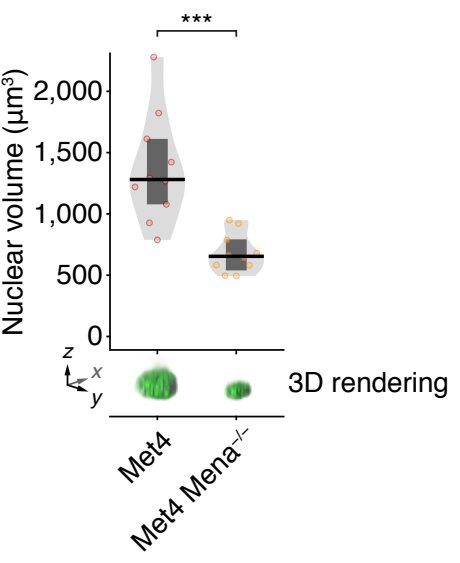

f Nuclear height

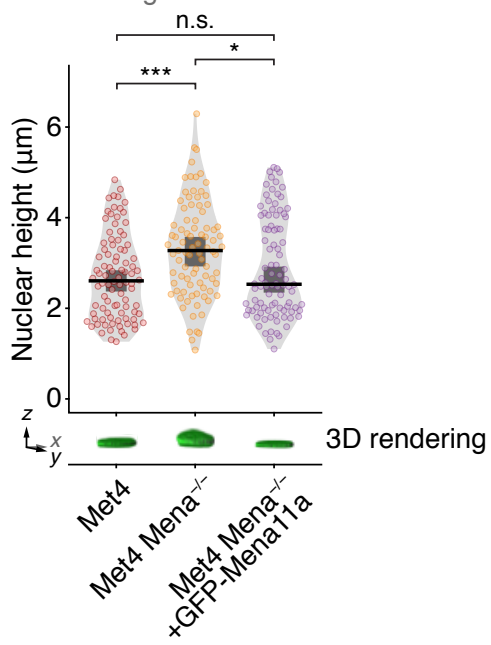

Figure 3. See next page for caption. 
bioRxiv preprint doi: https://doi.org/10.1101/2021.08.31.458340; this version posted August 31, 2021. The copyright holder for this preprint (which was not certified by peer review) is the author/funder, who has granted bioRxiv a license to display the preprint in perpetuity. It is made available under aCC-BY-NC-ND 4.0 International license.

Figure 3. Mena is required for nesprin-2 links to actin and lamin A/C and regulates nuclear morphology. (a, b) IP analyses of nesprin-2 protein complexes in Met4 cells, Mena-depleted Met 4 cells (Met4 Mena ${ }^{-/}$) and Met4 Mena ${ }^{-/-}$cells with Mena11a re-expressed (Met4 Mena ${ }^{-/}+$Mena11a). Actin (a) and lamin A/C (b) were detected by western blotting; respective densitometric intensities were normalised (norm.) to nesprin-2 and expressed relative (rel.) to Met 4 cells (right panels). Black bar, median; light grey box, range. ${ }^{* * \star} P<0.0001$, ${ }^{\star *} P<0.01,{ }^{\star} P<0.05$; n.s., not significant; Welch’s oneway ANOVA with two-stage Benjamini-Krieger-Yekutieli correction ( $n=6$ independent experiments) for a, one-way ANOVA with Tukey's correction ( $n=5$ independent experiments) for $\mathbf{b}$. (c) Spinning-disk confocal imaging of Met 4 cells and Met4 $\mathrm{Mena}^{-/-}$cells in 3D collagen matrix. Orthogonal projections $(x z)$ were extracted from 3D brightest-point projections. Nuclei were detected using DAPI; F-actin was detected using phalloidin. Inverted lookup tables were applied. Images are representative of two independent experiments. Scale bar, $20 \mu \mathrm{m}$. (d) Quantification of nuclear volume of cells in 3D matrix (see c). 3D volume renderings of exemplar nuclei detected using DAPI are displayed. Black bar, median; dark grey box, 95\% confidence interval; light grey silhouette, probability density. ${ }^{* \star *} P<0.001$; two-sided Welch's $t$-test $(n=10$ cells from $n=2$ independent experiments). (e) Spinning-disk confocal imaging of Met 4 cells, Met $4 \mathrm{Mena}^{-{ }^{-}}$cells and Met 4 $\mathrm{Mena}^{-1-}+$ GFP-Mena11a cells on 2D fibronectin matrix. Orthogonal projections were extracted from 3D brightest-point projections. Nuclei were detected using DAPI. Inverted lookup tables were applied. Images are representative of four independent experiments. Scale bar, $20 \mu \mathrm{m}$. (f) Quantification of nuclear height of cells on 2D matrix (see e). 3D volume renderings of exemplar nuclei detected using DAPI are displayed. Black bar, median; dark grey box, $95 \%$ confidence interval; light grey silhouette, probability density. ${ }^{* *} P<0.001,{ }^{\star} P<0.05$; n.s., not significant; Kruskal-Wallis test with Dunn's correction ( $n=92,87$ or 94 cells for Met4, Met4 Mena ${ }^{-/-}$and Met4 Mena ${ }^{-/-}+$GFP-Mena11a cells, respectively, from $n=4$ independent experiments). 
findings are consistent with Mena acting as a crucial facilitator/mediator of the interactions of nesprin-2 and the LINC complex, with actin and the nuclear lamina.

The interaction between the LINC complex and actin is reported to constrain nuclear shape in fibroblasts and carcinoma cells cultured in $2 \mathrm{D}^{59}$ and $3 \mathrm{D}$ matrices ${ }^{26}$. We examined nuclear morphology by immunofluorescence and found that, in 3D collagen matrices, nuclear volume was significantly reduced in Mena-depleted cells (Fig. 3c, d). This implicates Mena in control of nuclear architecture in 3D environments via regulation of the nesprin-2-actin association. On 2D surfaces, depletion of nesprin-2 or disruption of perinuclear actomyosin is reported to increase nuclear height ${ }^{60-62}$. We observed an increase in nuclear height in Mena-depleted cells grown on 2D fibronectin matrices, which was rescued by re-expression of Mena11a (Fig. 3e, f). Together, these data are consistent with (i) Mena being required for interaction between F-actin and the nucleus via the LINC complex, and (ii) Mena controlling nuclear architecture via the F-actin-LINC complex in Met4 cSCC cells.

\section{Mena controls emerin tyrosine phosphorylation and linked gene expression.}

LINC complex-actin cytoskeleton connections are known to mediate the transmission of tensile force generated by actomyosin contractility to the nucleus. Such nuclear force transmission was recently shown to induce tyrosine phosphorylation of the nucleoskeleton- and LINC complex-binding protein emerin, thereby mediating an adaptive nuclear-stiffening mechanoresponse to tension in fibroblasts and HeLa cells ${ }^{40}$. To validate whether emerin tyrosine phosphorylation constitutes a readout for nuclear mechanotransduction in Met 4 cSCC cells, we first disrupted actomyosin-based force contractility using the selective non-muscle myosin II inhibitor blebbistatin. Treatment with $10 \mu \mathrm{M}$ or $50 \mu \mathrm{M}$ blebbistatin for $2 \mathrm{~h}$ caused a dose-dependent reduction in emerin tyrosine phosphorylation (Fig. $4 \mathrm{a}$ ), consistent with previous studies ${ }^{40}$. Mena depletion also reduced emerin tyrosine phosphorylation in Met 4 cells, and this was rescued by re-expression of Mena11a, with higher overexpression of Mena11a leading to higher levels of emerin tyrosine phosphorylation (Fig. 4b). It is known that keratinocytes react to tension by increasing histone $\mathrm{H} 3$ trimethylation at lysine-27 $(\mathrm{H} 3 \mathrm{~K} 27 \mathrm{me} 3)^{63}$, and we found here that Mena depletion in Met4 cSCC cells resulted in a decrease in H3K27me3, which was rescued by re-expression of GFP-Mena11a (Supplementary Fig. 3a). Taken together, these data imply that Mena is required for relaying mechanical contractility cues to the nucleus by regulating the link between actin and the LINC complex, and that this has consequences for transcription regulatory mechanisms, such as histone $\mathrm{H} 3$ trimethylation.

Upon mechanical stress, emerin is tyrosine-phosphorylated at both Y74 and Y95 (ref. 40), which, together with Y59 phosphorylation, have been associated with a reduction in the binding of its LEM domain to the DNA-binding protein barrier-to-autointegration factor (BAF) in HeLa cells treated with pervanadate ${ }^{64}$. Emerin interaction with BAF mediates the recruitment of sections of chromatin known as lamina-associated domains (LADs) to the nuclear lamina, which has been associated with gene repression ${ }^{65}$. We therefore tested whether Mena-regulated changes in tyrosine phosphorylation of emerin could regulate the positioning of chromatin at the nuclear lamina. We performed multiplexed gene expression analysis of parental Met 4 cells and their Mena-depleted counterparts (Met4 $\mathrm{Mena}^{-/}$), digitally profiling the expression of 770 genes associated with cancer progression (Fig. 4c, Supplementary Fig. 3b, Supplementary Table 3). We found that Mena depletion caused a statistically significant change in the expression of 48 of the profiled cancer-associated genes (Fig. 4d, e, Supplementary Fig. 3c), which were broadly associated with regulation of cell adhesion and migration, ECM organisation and the immune response (Fig. 4d, Supplementary Fig. 3d, Supplementary Table 4). Of the dysregulated genes, the expression of 15 was significantly reduced by at least two-fold upon Mena depletion (Fig. 4e, Supplementary Table 4). Among these were the genes encoding fibronectin (FN1) and a5 integrin (ITGA5) (Fig. 4d, e, Supplementary Fig. 3d), consistent with a previously published study showing 
bioRxiv preprint doi: https://doi.org/10.1101/2021.08.31.458340; this version posted August 31, 2021. The copyright holder for this preprint (which was not certified by peer review) is the author/funder, who has granted bioRxiv a license to display the preprint in perpetuity. It is made available under aCC-BY-NC-ND 4.0 International license.

a Emerin tyrosine phosphorylation detection

Emerin (pY) rel. intensity (norm. to total emerin)
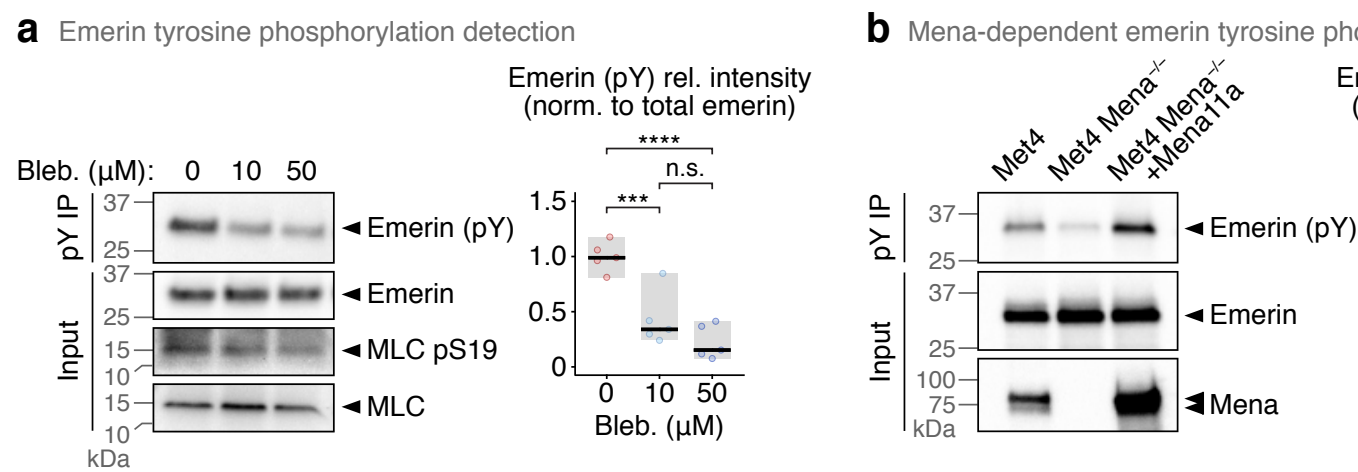

horylation

Emerin (pY) rel. intensity (norm. to total emerin)

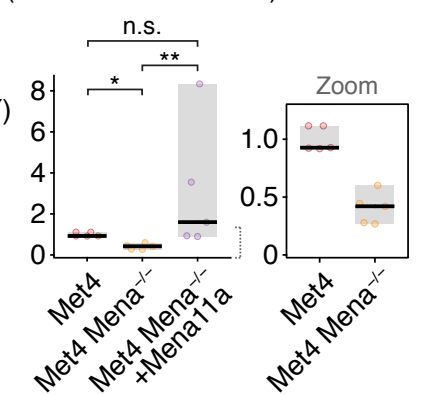

Multiplexed gene expression analysis

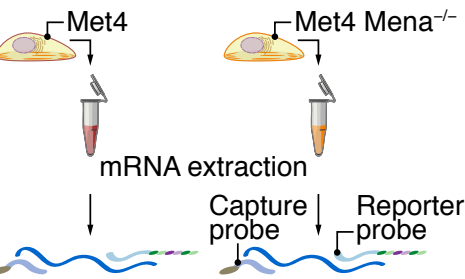

Barcoded probe-pair hybridisation

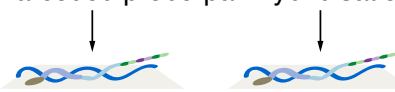

Purification and immobilisation

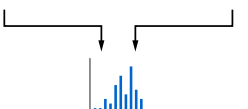

Quantitative nCounter analysis
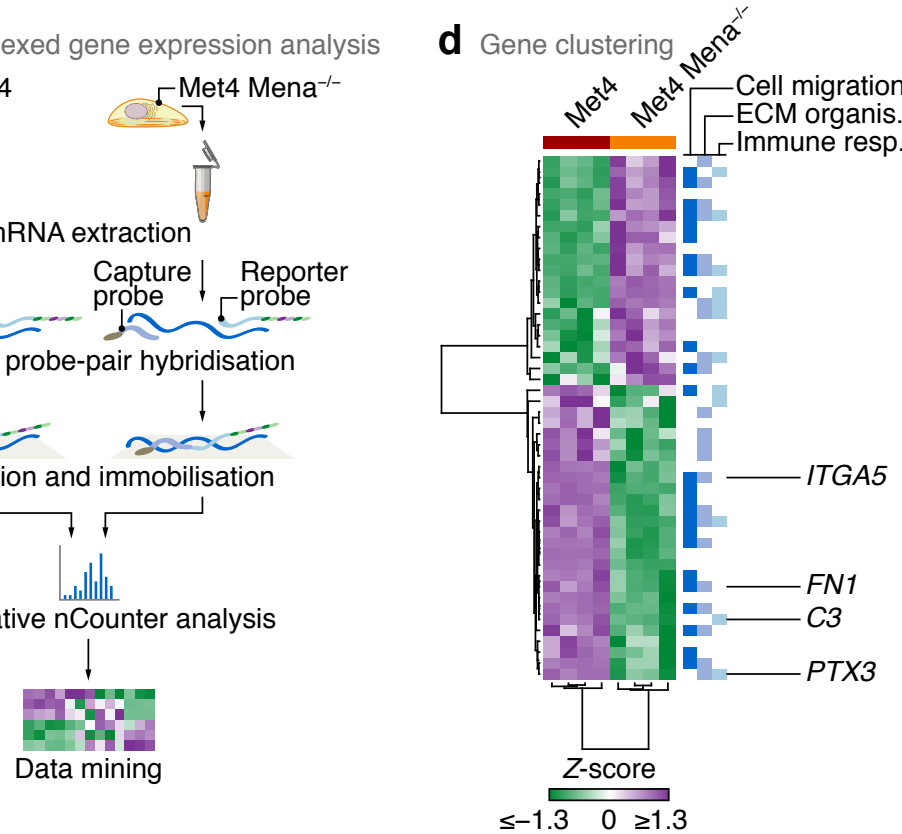

e Transcriptional regulation

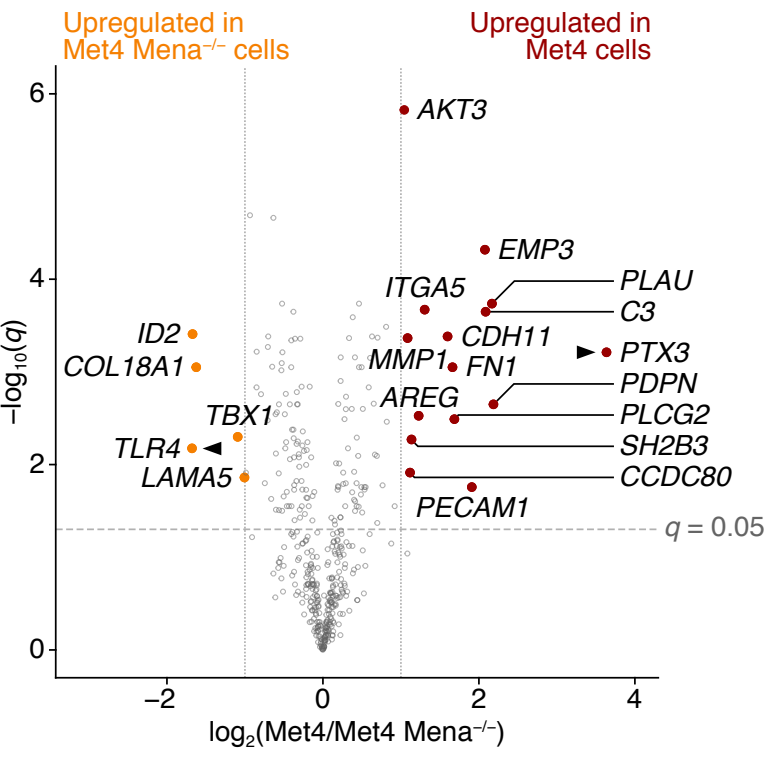

f Nuclear lamina recruitment analysis

g РTXЗ enhancer repositioning

Dam-lamin B1
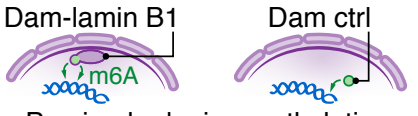

Proximal adenine methylation

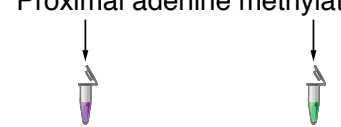

gDNA extraction and digestion

Methylated gDNA amplification

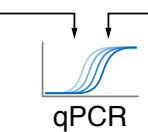

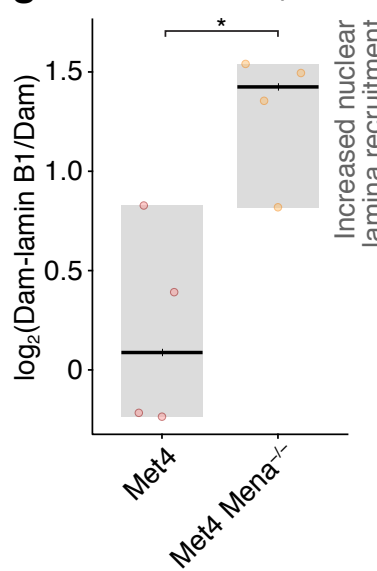

h

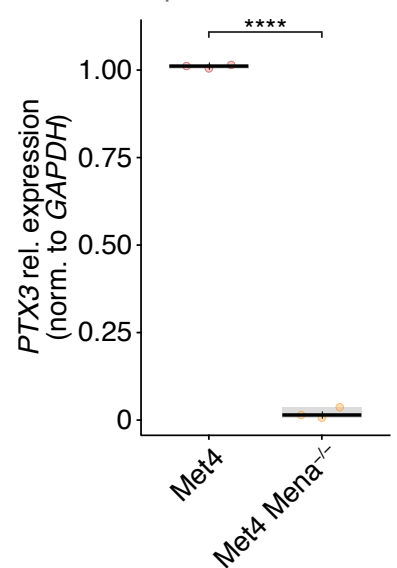

i cSCC gene expression

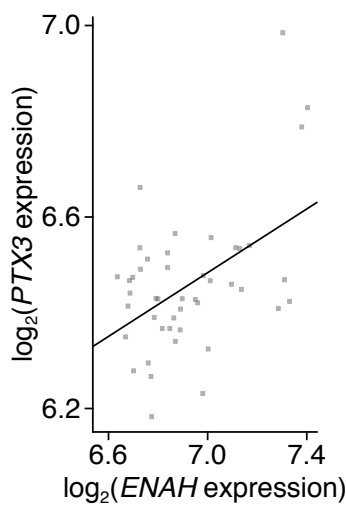

Figure 4. See next page for caption. 
bioRxiv preprint doi: https://doi.org/10.1101/2021.08.31.458340; this version posted August 31, 2021. The copyright holder for this preprint (which was not certified by peer review) is the author/funder, who has granted bioRxiv a license to display the preprint in perpetuity. It is made available under aCC-BY-NC-ND 4.0 International license.

Figure 4. Mena loss suppresses emerin tyrosine phosphorylation and regulates PTX3 expression. (a) Emerin tyrosine phosphorylation after treatment of cells with blebbistatin. Met 4 cells were treated with 0 (vehicle control), 10 or $50 \mu \mathrm{M}$ blebbistatin for $2 \mathrm{~h}$. Emerin tyrosine phosphorylation was detected by phospho-tyrosine (pY) IP followed by western blotting for emerin; densitometric intensities were normalised to total emerin (lysate) and expressed relative to vehicle control (right panel). Black bar, median; light grey box, range. ${ }^{* * *} P<0.0001$, ${ }^{\star * \star} P<0.001$; n.s., not significant; one-way ANOVA with Tukey's correction ( $n=5$ independent experiments). MLC, myosin light chain. (b) Emerin tyrosine phosphorylation in Met4, Met4 Mena ${ }^{-/-}$and Met4 $\mathrm{Mena}^{-1-}+$ Mena1la cells was detected and quantified as for a. Inset (right panel) shows zoom of quantification of Met 4 and Met $4 \mathrm{Mena}^{-/-}$cells for clarity. ${ }^{\star \star} P<0.01,{ }^{\star} P<0.05$; n.s., not significant; Kruskal-Wallis test with two-stage Benjamini-Krieger-Yekutieli correction ( $n=5$ independent experiments). (c) Workflow for multiplexed digital quantification of the expression of 770 genes associated with cancer progression. Dark blue strand, messenger RNA (mRNA). (d) Hierarchical cluster analysis of cancer progression genes significantly differentially regulated between Met 4 and Met 4 Mena $^{-/}$cells $(q<0.05$, two-sided $t$-test with Benjamini-Hochberg correction; $n=4$ independent biological replicates). Genes associated with cell migration, ECM organisation (ECM organis.) or the immune response (immune resp.) are indicated (blue bars). (e) Volcano plot of cancer progression genes. Genes significantly differentially regulated between Met 4 and Met $4 \mathrm{Mena}^{-1-}$ cells and upregulated by at least two-fold are labelled. The most differentially regulated genes in Met 4 and Met $4 \mathrm{Mena}^{-1-}$ cells, respectively, are indicated with black arrowheads. Genes expressed at levels below the background threshold of 20 counts were omitted. (f) Workflow for quantification of recruitment of specific regions of chromatin to the nuclear lamina by DamID. DNA adenine methyltransferase (Dam) tethered to lamin B1 or untethered Dam control (ctrl) was expressed in Met4 and Met4 Mena ${ }^{-/-}$ cells. Dark blue strands, genomic DNA (gDNA); green arrows, adenine methylation (m6A) in GATC motifs proximal to Dam. (g) Nuclear lamina association of PTX3 quantified by lamin B1 DamID-qPCR. qPCR primers amplified the highestscoring putative enhancer region associated with $P T X 3$ (GeneHancer identifier GH03J157436). ${ }^{\star} P<0.05$; two-sided Student's $t$-test ( $n=4$ independent experiments). (h) Quantification of PTX3 expression in Met 4 and Met $4 \mathrm{Mena}^{-1-}$ cells by RT-qPCR. Gene expression was normalised to GAPDH and expressed relative to Met 4 cells. Black bar, median; light grey box, range. ${ }^{* * *} P<0.0001$; two-sided Student's $t$-test ( $n=3$ independent experiments). (i) Correlation of ENAH and PTX3 expression determined by analysis of microarray data from patient-derived cSCC cell lines (GEO series accession identifier GSE98767). Pearson correlation coefficient $=0.487 ; P=6.87 \times 10^{-4}(n=45$ samples from $n=3$ independent replicates). 
that ENAH expression correlates with FN1 and ITGA5 expression ${ }^{66}$. Mena loss also resulted in downregulation in the expression of genes that encode immunomodulatory secretory proteins that are part of the complement system, such as PTX3 and C3 (Fig. 4d, e, Supplementary Fig. 3d), suggesting that Mena may regulate immunosurveillance via the tumour-associated microenvironment.

We next addressed whether the Mena-dependent effects on some Mena-dependent transcriptional changes correlated with recruitment of LADs to the nuclear lamina that is associated with reduced tyrosine phosphorylation of emerin upon Mena depletion. We used DNA adenine methyltransferase identification (DamID) to map chromatin interactions with the nuclear lamina across the cSCC genome. Parental Met4 cells and their Mena-deficient counterparts were transduced with Dam or with Dam fused to nuclear lamina protein lamin B1 (Fig. 4f, Supplementary Fig. 3e). Adenines in DNA regions in close proximity to lamin B1, and likely proximal to emerin, were thus methylated (m6A) in GATC motifs by the tethered Dam as described previously ${ }^{67}$. The genomic fragments flanked by methylated GATC motifs were sequenced and the data converted to ratios of Dam-lamin B1 versus untethered Dam control normalised reads to produce genomewide nuclear lamina association profiles (Supplementary Fig. 3e). Upon Mena depletion, we observed a significant lamin B1 DamID peak (FDR 1\%) at a region of chromosome 3 that corresponds to a predicted enhancer for PTX3 (highest-scoring cis-regulatory element by GeneHancer analysis) ${ }^{68}$, which exhibited increased association with the nuclear lamina in Mena-deficient cells compared to parental Met4 cells (Supplementary Fig. 3f). Analysis of DamID fragments by qPCR confirmed an increase in nuclear laminaassociated chromatin spanning the PTX3 enhancer locus in Mena-deficient cells (Fig. 4g), implying that the $P T X 3$ enhancer is recruited to the nuclear lamina upon Mena depletion. Enhancers sequestered to the nuclear lamina can be associated with their inactivation ${ }^{69}$. Our findings are therefore consistent with the observation that PTX3 is significantly downregulated in Mena-depleted Met 4 cells, as we showed by multiplexed gene expression analysis (Fig. 4d, e, Supplementary Fig. 3d) and confirmed by RT-qPCR (Fig. 4h), via repositioning of particular LADs to the nuclear periphery. Moreover, previous studies have shown that PTX3 expression is inducible by the interleukin IL- $1 \beta$. The latter is downregulated in Mena-deficient cells, as determined by gene expression analysis (Supplementary Fig. 3d), suggesting another regulatory layer by which Mena could regulate PTX 3 expression. To confirm that Mena does commonly regulate the expression of PTX3 in cSCC, we analysed publicly available microarray transcriptomics data from patient-derived cSCC samples (GEO series accession identifier GSE98767) ${ }^{70}$ and found that ENAH and PTX3 expression are positively correlated (Pearson correlation coefficient $=0.487, P=6.87 \times 10^{-4}$ ) in human cSCC (Fig. $4 \mathrm{i}$ ).

Taken together, our results imply that Mena regulates the expression of genes, at least in part, through its interaction with nesprin-2, which links the actomyosin contractile apparatus of the cell, via the LINC complex, to the nucleus (Fig. 5). This, in turn, elicits changes in emerin tyrosine phosphorylation at the nuclear lamina, and we identified PTX3 as a gene whose transcription is regulated by Mena as a result of altered chromatin organisation (Fig. 5).

\section{DISCUSSION}

Mena is an actin regulatory protein, mostly defined at actin-rich structures, such as lamellipodia, filopodia, focal adhesions and cell-cell contacts, as well as stress fibres, along which it forms periodic puncta ${ }^{71}$. Here, we show that Mena is the most significantly enriched actin-binding protein in the adhesome of metastatic Met 4 cSCC cells when compared to their non-metastatic counterparts from the same patient. Using a network analysis approach, we found that Mena was part of a functional adhesome module consisting of F-actinbinding and regulatory proteins in the metastatic cells. Subnetwork association of Mena and the LINC complex component nesprin-2 led us to uncover a novel function of Mena at the perinucleus, whereby it interacts with the C-terminal SRs of nesprin-2 and potentiates F-actin binding to nesprin-2 (Fig. 5). This, in line with other 
bioRxiv preprint doi: https://doi.org/10.1101/2021.08.31.458340; this version posted August 31, 2021. The copyright holder for this preprint (which was not certified by peer review) is the author/funder, who has granted bioRxiv a license to display the preprint in perpetuity. It is made available under aCC-BY-NC-ND 4.0 International license.

a Perinuclear Mena

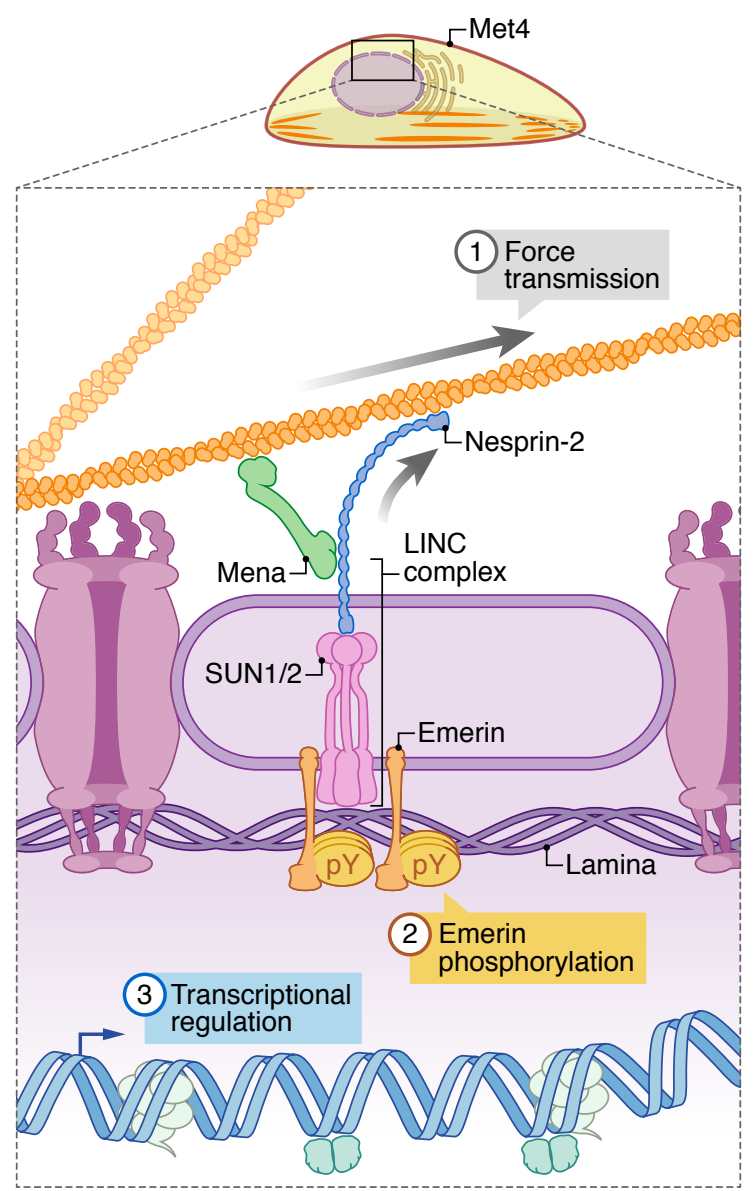

b Mena loss

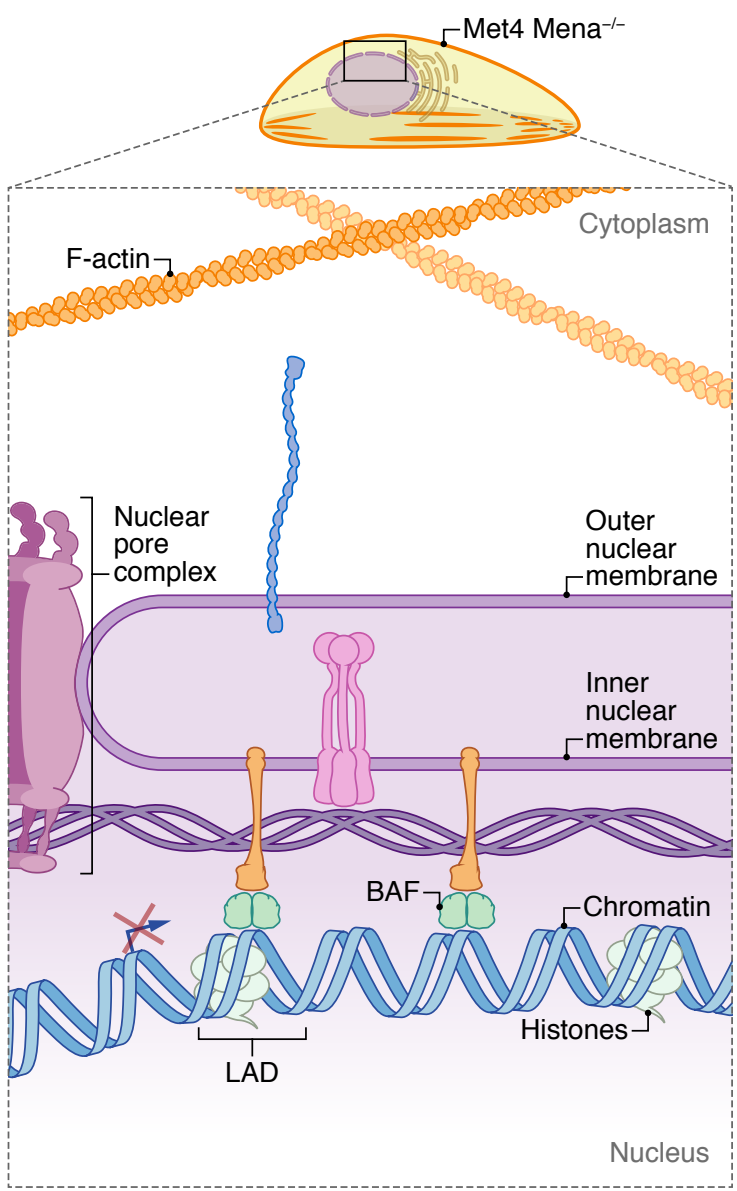

Figure 5. Model of the proposed role for Mena at the nuclear membrane. (a) Mena interacts with nesprin-2 at the outer nuclear membrane via the C-terminal SRs of nesprin-2. This potentiates the interactions of nesprin-2 with F-actin and lamin $\mathrm{A} / \mathrm{C}$, permitting force transmission from the actin cytoskeleton to the nuclear lamina (1). Actomyosin-based force maintains emerin tyrosine phosphorylation. Emerin tyrosine phosphorylation has been reported to prevent its LAP2emerin-MAN1 (LEM) domain from binding to the DNA-binding protein barrier-to-autointegration factor (BAF), limiting chromatin repositioning to the nuclear lamina (2). This proposed mechanism allows genomic loci to favour chromatin decondensation and expression of specific genes, including, in metastatic Met 4 cSCC cells, those involved in cancer progression, such as genes associated with cell adhesion and migration (e.g. ITGA5, PLAU), ECM organisation (e.g. FN1, $M M P 1)$ and the immune response (e.g. PTX3, C3) (3). (b) Mena loss decouples F-actin from nesprin-2, diminishing force transduction to the nuclear envelope via nesprin-2. Mena loss thus results in a reduction of emerin tyrosine phosphorylation, which has been reported to promote BAF binding to the emerin LEM domain, and enhances recruitment of heterochromatic lamina-associated domains (LADs) to the nuclear periphery. Chromatin repositioning is associated with transcriptional silencing of genes with regulatory elements in specific LADs, such as the immunomodulatory gene PTX3 in cSCC cells. 
observations ${ }^{26,38,55}$, supports the view that actin-binding proteins may mediate the interaction of nesprin SRs with $\operatorname{actin}^{72}$, and we show here that Mena is one of these. We also found that Mena regulates nuclear morphology in Met 4 cells, consistent with its function of mediating the interaction of actin with nesprin-2. By modulating LINC complex interactions, Mena controls phosphorylation of the nuclear membrane protein emerin, repositioning of chromatin at the nuclear periphery and the regulation of some genes involved in tumour progression (proposed model in Fig. 5). How Mena is transported to the perinuclear region to elicit these functions, and the nature of common and distinct pools of Mena, is not known.

Delineating a novel role for Mena at the perinucleus, we show here that Mena is required for the mechanoresponse of emerin (Fig. 5). Emerin is a member of the LEM domain protein family, forming an extensive interaction network with nuclear lamina and nucleoplasmic proteins. We found that Mena depletion results in the reduction of emerin tyrosine phosphorylation and an increased association of PTX3 with the nuclear lamina. Interestingly, emerin phosphorylation can also lead to remodelling of its interaction network at the nuclear lamina ${ }^{73}$. For instance, the interaction of emerin with histone deacetylase 3 (HDAC3) results in increased HDAC3 deacetylase activity, which is associated with gene repression ${ }^{74}$. Emerin phosphorylation is postulated to affect its interaction with HDAC3 at the nuclear lamina, thereby modulating mechanoresponsive gene expression $^{73}$, and we found that Mena loss results in a reduction of H3K27me3, a histone modification associated with gene repression. A previous study has shown that mechanical strain induces H3K27me3 and reduces active RNA polymerase II 'occupancy' in keratinocytes, and this is dependent on a perinuclear emerinnon-muscle myosin II-F-actin axis ${ }^{63}$. Further investigating the role of Mena in this emerin-actomyosin axis may provide insights into the mechanism of Mena-dependent H3K27me3 modification.

We found that Mena status controls gene expression, an example of which is Mena-regulated expression of PTX3. Mena also regulates the association of a PTX3 enhancer with the nuclear lamina and the expression of the gene encoding IL- $1 \beta$, an inducer of PTX3 expression. Tumour-promoting and tumoursuppressive roles for PTX3 have been described, while elevated levels of PTX3 correlate with either poor prognosis or grade of malignancy in several cancers ${ }^{75}$. Though the role of PTX3 in the context of cSCC is unknown, regulation of the expression of PTX3, and other immune response genes, by perinuclear activities of Mena supports observations that focal adhesion proteins have nuclear or nucleus-associated functions in regulating immunomodulatory proteins ${ }^{31}$. Indeed, we have demonstrated previously that nuclear FAK induces the expression of proinflammatory cytokines and chemokines, including IL-33 and CCL5, which inhibit antitumour immunity and drive tumour growth in vivo ${ }^{76,77}$.

In conclusion, we here report systems-level changes to the adhesome of cSCC cells that have undergone metastasis, adding to our understanding of the putative molecular mechanisms through which adhesion protein networks may drive cancer progression. This work establishes a novel connection between the adhesome component Mena and the LINC complex, through which Mena regulates actin-nuclear lamina interactions, nuclear architecture and chromatin organisation at the nuclear periphery, fine-tuning cancer gene expression, as demonstrated by the immunomodulatory gene PTX3, in metastatic keratinocytes.

\section{METHODS}

Cell culture. Human cSCC Met1 and Met4 cells (malignant keratinocytes) were cultured as described previously ${ }^{78,79}$ in RM+ medium consisting of high-glucose Dulbecco's modified Eagle's medium (DMEM; Sigma-Aldrich, \#D6546) and Ham's F-12 nutrient mixture (Life Technologies, \#21765-037) (3:1 DMEM:Ham's F-12 ratio) supplemented with $10 \%$ (v/v) foetal bovine serum (FBS; Life Technologies, \#10270106), $0.4 \mu \mathrm{g} / \mathrm{ml}$ hydrocortisone (STEMCELL Technologies, \#7904), $3 \mathrm{mM} \mathrm{L-glutamine} \mathrm{( \# 200049),} 5 \mu \mathrm{g} / \mathrm{ml}$ transferrin (\#T2252), $1 \times 10^{-10} \mathrm{M}$ cholera toxin (\#C8052), $10 \mathrm{ng} / \mathrm{ml}$ epidermal growth factor (\#E2644), $5 \mu \mathrm{g} / \mathrm{ml}$ insulin (\#19278) and $2 \times 10^{-11} \mathrm{M}$ liothyronine (all Sigma-Aldrich, \#T6397). HEK293T cells (a gift from Noor 
Gammoh, University of Edinburgh, Edinburgh UK) were cultured in high-glucose DMEM supplemented with $10 \%(\mathrm{v} / \mathrm{v})$ foetal bovine serum. Cells were grown at $37^{\circ} \mathrm{C}$ in humidified $5 \%(\mathrm{v} / \mathrm{v}) \mathrm{CO}_{2}$. Cells routinely tested negative for mycoplasma.

For 3D culture in collagen, Met 1 or Met 4 cells were trypsinised and collected by centrifugation at 200 $\times g$ for 5 min. Cells $\left(5 \times 10^{4}\right)$ were resuspended in $2-3 \mathrm{mg} / \mathrm{ml}$ rat-tail collagen I in RM+ medium and seeded on 23-mm FluoroDish cell culture dishes (World Precision Instruments). The collagen with embedded cells was allowed to set at $37^{\circ} \mathrm{C}$ until collagen contraction was observed $(\sim 30 \mathrm{~min})$. Another aliquot of collagenembedded cells was added to the contracted matrix and allowed to set at $37^{\circ} \mathrm{C}$. Collagen-embedded cells were then incubated in RM+ medium.

Cell line generation and transfection. Met 1 cells were derived from primary cSCC and Met 4 cells from distant metastatic cSCC from the same immunosuppressed patient as described previously ${ }^{43,78}$. Ethical approval (REC reference 08/S1401/69, 5 November 2008) for this study was obtained from the East London and City Health Authority local ethics committee, and the study was conducted according to the Declaration of Helsinki Principles.

Met4 $\mathrm{Mena}^{-/-}$cells were generated by CRISPR/Cas9 technology. Guide oligonucleotides targeting exon 2 of ENAH (forward insert, 5'-TTTCTTGGCTTTATATATCTTGTGGAAAGGACGAAACACCGGAGATGCTAGACAGGTGTA-3'; reverse insert, 5'-GACTAGCCTTATTTTAACTTGCTATTTCTAGCTCTAAAACTACACCTGTCTAGCATCTCC-3') containing an AflII restriction site and protospacer adjacent motif were designed and synthesised (Integrated DNA Technologies). The gRNA cloning vector (Addgene, \#41824) was linearised by AflII digestion, and guide oligonucleotides were annealed to the linearised gRNA cloning vector by Gibson Assembly. Constructs were transformed into chemically competent DH5a cells and selected on agar plates containing kanamycin. Positive gRNA guides were identified by sequencing. Met 4 cells were cotransfected with Mena gRNA and hCas9 (Addgene, \#41824) using the Amaxa human keratinocyte Nucleofector kit (Lonza, \#VPD-1002) according to manufacturer's instructions, with a Nucleofector (Lonza) set to programme T-024. Three rounds of nucleofection were performed, allowing cells to recover and grow to $60 \%$ confluency between nucleofection rounds.

Cells were transiently transfected with GFP-nesprin-2G(SR49-56) (the eight C-terminal nesprin-2 SRs 49-56), GFP-nesprin-2G $\Delta$ SR3-50 (containing SRs 51-56 of nesprin-2; based on the nesprin-2 giant isoform, nesprin-2G) ${ }^{26}$, pEGFP-C1 or pcDNA-Mena11a (a gift from Francesca Di Modugno, Regina Elena National Cancer Institute, Rome, Italy). All GFP tags used were EGFP. Cells were transiently transfected with constructs using the X-tremeGENE HP DNA transfection reagent (Roche) according to manufacturer's instructions. Briefly, vectors were prepared (2:1 transfection reagent:DNA ratio) and incubated for at least 30 min prior to transfection of $2 \times 10^{6}$ cells seeded on fibronectin-coated cell culture dishes. Cells were used for experiments $60 \mathrm{~h}$ after transfection.

GFP-Mena1la was expressed in Met4 Mena $^{-/-}$cells as described previously ${ }^{77}$ using pMSCV-EGFPMena11a (a gift from Frank Gertler, Massachusetts Institute of Technology, Cambridge, MA USA). Phoenix amphoteric cells were transfected with pMSCV-EGFP-Menal la using Lipofectamine 2000 (Life Technologies) according to manufacturer's instructions. Briefly, $18 \mathrm{~h}$ after transfection, viral production was induced with fresh medium supplemented with $20 \%$ (v/v) FBS. Virus was harvested 48 and $72 \mathrm{~h}$ after transfection, and the viral supernatant was filtered through a $0.45-\mu \mathrm{m}$ Millex-HA filter (Millipore). Filtered viral supernatant was supplemented with $5 \mu \mathrm{g} / \mathrm{ml}$ polybrene (Millipore) and added to Met $4 \mathrm{Mena}^{-/-}$cells for $24 \mathrm{~h}$. Two rounds of transduction were performed. GFP-Mena11a-expressing cells were selected by fluorescence-activated cell sorting. 
Blebbistatin treatment. Cells were treated with $10 \mu \mathrm{M}$ and $50 \mu \mathrm{M}$ blebbistatin (in DMSO; Calbiochem) or DMSO (vehicle control) for $2 \mathrm{~h}$ at $37^{\circ} \mathrm{C}$ in humidified $5 \%(\mathrm{v} / \mathrm{v}) \mathrm{CO}_{2}$.

Immunoprecipitation and pulldown. For nesprin-2 immunoprecipitation (IP), cells were washed twice with ice-cold PBS and lysed in NETN lysis buffer (100 mM NaCl, $20 \mathrm{mM}$ Tris- $\mathrm{HCl}$, pH 7.5, $0.5 \mathrm{mM}$ EDTA, 0.5\% (v/v) NP-40) supplemented with protease inhibitors. Cells were homogenised by 15 strokes through a $23 \mathrm{G}$ syringe needle. Homogenate was clarified by centrifugation at $16,000 \times g$ for $10 \mathrm{~min}$ at $4^{\circ} \mathrm{C}$. Supernatant $(1 \mathrm{mg}$ protein) was incubated overnight with rotation at $4^{\circ} \mathrm{C}$ with either nesprin-2 antibody (Abcam, \#ab217057) or anti-rabbit IgG (Cell Signaling Technology, \#2729) and Protein G Dynabeads (Invitrogen, \#10003D). Beads were washed three times with lysis buffer, and isolated immune complexes were eluted with Laemmli sample buffer for $10 \mathrm{~min}$ at $95^{\circ} \mathrm{C}$.

For phosphotyrosine (PY20) IP, immune complexes were isolated as above, except cells were lysed in RIPA buffer (50 mM Tris- $\mathrm{HCl}, \mathrm{pH} 7.5,150 \mathrm{mM} \mathrm{NaCl}, 1 \%$ (v/v) Triton X-100, 0.5\% (w/v) sodium deoxycholate) supplemented with protease and phosphatase inhibitors. Supernatant was incubated with PY20 anti-phosphotyrosine antibody (BD Transduction, \#610000) or, for blebbistatin treatment experiments, PY20 antibody coupled to M-270 Epoxy Dynabeads (Invitrogen, \#14301) according to manufacturer's instructions.

GFP-Trap pulldown was performed as described previously ${ }^{26}$ with modifications. Briefly, cells were treated with $3 \mathrm{mM}$ dimethyl 3,3'-dithiobispropioimidate (DTBP; Thermo Fisher Scientific, \#20665) for $5 \mathrm{~min}$ at $37^{\circ} \mathrm{C}$, which was then quenched with $200 \mathrm{mM}$ Tris- $\mathrm{HCl}$ (pH 7.8) for $10 \mathrm{~min}$ at room temperature. Cells were lysed in RIPA buffer as above, except lysates were sonicated after homogenisation. Supernatant was incubated with GFP-Trap magnetic agarose (ChromoTek).

Western blotting. Protein concentration was measured by Pierce BCA protein assay (Thermo Fisher Scientific). Protein was supplemented with Laemmli sample buffer to a final concentration of $0.5-1.0 \mathrm{mg} / \mathrm{ml}$ and boiled for $10 \mathrm{~min}$ at $95^{\circ} \mathrm{C}$. Proteins were resolved by SDS-polyacrylamide gel electrophoresis using $4-15 \%$ Mini-PROTEAN TGX gels (Bio-Rad) or, to resolve anti-nesprin-2-reactive bands, 3-8\% Tris-acetate gels (Life Technologies. Proteins were transferred to nitrocellulose membrane by semi-dry transfer (Trans-Blot Turbo transfer system; Bio-Rad) or, to transfer nesprin-2, by wet transfer in Tris-glycine buffer (Bio-Rad) supplemented with $15 \%(\mathrm{v} / \mathrm{v})$ methanol. Membranes were blocked with $5 \%(\mathrm{w} / \mathrm{v})$ milk or $5 \%(\mathrm{w} / \mathrm{v})$ bovine serum albumin (BSA) in Tris-buffered saline (TBS). Membranes were probed with the following primary antibodies (all Cell Signaling Technology, diluted 1:1,000, unless otherwise stated): anti- $\alpha \mathrm{V}$ integrin (Abcam, \#ab179475), anti-actin (\#4970), anti-cytochrome c oxidase subunit 4 (\#11967), anti-emerin (\#30853), antiFAK (\#3285), anti-FAK pY397 (\#3283), anti-GAPDH (\#5174), anti-GFP (BioVision, \#3999-100), antiH3K27me3 (\#9733), anti-histone H3 (\#4499), anti-lamin A/C (\#2032), anti-Mena (Atlas, \#HPA028448), antimyosin light chain 2 (\#8505), anti-myosin light chain 2 pS19 (\#3675), anti-nesprin-2 (Abcam, \#ab217057). Membranes were incubated with anti-rabbit or anti-mouse streptavidin-conjugated horseradish peroxidase secondary antibodies (Cell Signaling Technology) and visualised using a ChemiDoc MP Imaging System (Bio$\mathrm{Rad})$.

Immunofluorescence microscopy. For cells on 2D substrate, cells were seeded on fibronectin-coated coverslips and allowed to spread overnight. Cells were fixed with $4 \%(\mathrm{w} / \mathrm{v})$ paraformaldehyde for $15 \mathrm{~min}$, washed twice with PBS and incubated in $0.1 \mathrm{M}$ glycine for $5 \mathrm{~min}$. Cells were permeabilised with $0.2 \%(\mathrm{v} / \mathrm{v})$ Triton X-100 in PBS for 5 min, washed twice with PBS and blocked with 2\% (w/v) BSA in PBS for $1 \mathrm{~h}$. Cells were incubated with anti-Mena (Merck Millipore, \#MAB2635) or anti-nesprin-2 (Abcam, \#ab204308), diluted 1:200 in $2 \%(\mathrm{w} / \mathrm{v}) \mathrm{BSA}$ in PBS, overnight at $4^{\circ} \mathrm{C}$. Cells were washed six times with PBS and incubated with 
$\mathrm{F}(\mathrm{ab})_{2}$ secondary antibody conjugated to Alexa Fluor 594 (Thermo Fisher Scientific) or ATTO 647N (Rockland Immunochemicals) and phalloidin conjugated to Alexa Fluor 488 (Thermo Fisher Scientific), diluted 1:400 (1:800 for phalloidin) in $0.05 \%(\mathrm{v} / \mathrm{v})$ Tween 20 in PBS, for $45 \mathrm{~min}$ in the dark. Cells were washed six times with PBS, incubated with $4 \%$ (w/v) paraformaldehyde for $5 \mathrm{~min}$, washed with PBS and incubated with $0.1 \mathrm{M}$ glycine for $10 \mathrm{~min}$. Coverslips were mounted with anti-fade Fluoroshield containing DAPI (Sigma-Aldrich).

For cells in 3D collagen matrices, matrix-embedded cells were fixed with formaldehyde-PIPES buffer (4\% (w/v) formaldehyde, $100 \mathrm{mM}$ PIPES, pH 6.8, $10 \mathrm{mM}$ EGTA, pH 8.0, $1 \mathrm{mM} \mathrm{MgCl}_{2}, 0.2 \%$ (v/v) Triton X100) for $30 \mathrm{~min}$ and permeabilised with $0.2 \%(\mathrm{v} / \mathrm{v})$ Triton X-100 in TBS (TBS-TX) for $30 \mathrm{~min}$. Autofluorescence of the collagen matrix was quenched with two 10 -min washes with $0.5 \mathrm{mg} / \mathrm{ml} \mathrm{NaBH} \mathrm{H}_{4}$. Cells were blocked with $2 \%(\mathrm{w} / \mathrm{v})$ BSA in TBS-TX for $4 \mathrm{~h}$. Cells were incubated with anti-Mena (Atlas, \#HPA028448), diluted 1:200 in 2\% (w/v) BSA in TBS-TX, overnight at $4^{\circ} \mathrm{C}$. Cells were washed six times with TBS-TX and incubated with $\mathrm{F}(\mathrm{ab})_{2}$ secondary antibody conjugated to Alexa Fluor 594 and phalloidin conjugated to ATTO 647N (Sigma-Aldrich), diluted 1:400 in TBS-TX. Cells were washed six times with TBSTX, and coverslips mounted on collagen matrices with anti-fade Fluoroshield containing DAPI.

Spinning-disk confocal images were acquired on a Dragonfly multimodal imaging platform (Andor Technology) with 405-, 488-, 561- and 637-nm excitation laser lines using a 100× immersion objective. Data were collected in spinning-disk $25-\mu \mathrm{m}$ pinhole mode on a iXon 888 EMCCD camera (Andor Technology) using $1 \times 1$ binning and frame averaging of four. Confocal images were acquired with $z$-step size of $0.5 \mu \mathrm{m}$ using a piezo positioning system (Mad City Labs). For comparative experiments, images were collected with the same settings for all cell lines.

SIM images were acquired on an N-SIM super-resolution microscope (Nikon Instruments) with 488, 561- and 640-nm excitation laser lines using a 100× 1.49NA lens and refractive index-matched immersion oil (Nikon Instruments). Cells were imaged using a DU-897X-5254 camera (Andor Technology). Image $z$-step size was set to $0.120 \mu \mathrm{m}$ as recommended by the manufacturer's software. For each focal plane, 15 images ( 5 phases, 3 angles) were captured. SIM image processing, reconstruction and analysis were carried out using the N-SIM module in NIS-Elements Advanced Research software (version 4.6) (Nikon Instruments).

For nuclear morphology analyses, regions of interest (nuclei) were generated on the basis of DAPI staining. Nuclear height was measured from the minimum length of the object-orientated bounding box that fully enclosed the nucleus, implemented in Imaris (version 9.2.0) (Oxford Instruments). Nuclear volume was measured and rendered in Fiji (version 1.52p) ${ }^{80}$. DAPI-stained images were background subtracted using a sliding paraboloid set to 50 pixels and thresholded using the Moments method. Voxel measurements were limited to the thresholded region.

Adhesome isolation. IAC isolation was performed as described previously ${ }^{11,12}$. Briefly, $3 \times 10^{6} \mathrm{cSCC}$ cells were seeded on $15-\mathrm{cm}$ cell culture dishes coated with $10 \mu \mathrm{g} / \mathrm{ml}$ human fibronectin (Corning, \#356008) and incubated overnight. Cells were crosslinked with 3 mM DTBP in DMEM/Ham's F-12 nutrient mixture (1:1 DMEM:Ham's F-12 ratio) for $5 \mathrm{~min}$ at $37^{\circ} \mathrm{C}$, which was then quenched with $200 \mathrm{mM}$ Tris- $\mathrm{HCl}$ (pH 6.8) for 5 $\mathrm{min}$ at room temperature. Cells were washed with $200 \mathrm{mM}$ Tris- $\mathrm{HCl}$ (pH 6.8) and lysed with ice-cold IAC extraction buffer $\left(0.05 \%(\mathrm{w} / \mathrm{v}) \mathrm{NH}_{4} \mathrm{OH}, 0.5 \%(\mathrm{w} / \mathrm{v})\right.$ Triton X-100 in PBS). Cell bodies were removed by hydrodynamic pressure applied with a Waterpik device (Waterpik Ultra Water Flosser WP-120), and IAC proteins were isolated using adhesion recovery buffer $(125 \mathrm{mM}$ Tris- $\mathrm{HCl}, \mathrm{pH} 6.8,1 \%$ (w/v) SDS, $150 \mathrm{mM}$ dithiothreitol). Isolated IACs were analysed by western blotting (see above) or processed for LC-MS/MS analysis (see below).

For proteomic analysis, isolated IACs were precipitated with acetone as described previously ${ }^{12}$. Protein pellets were resuspended in $0.2 \%(\mathrm{w} / \mathrm{v})$ RapiGest (Waters) for $2 \mathrm{~h}$, incubated at $90^{\circ} \mathrm{C}$ for $10 \mathrm{~min}$ and subjected 
to in-solution tryptic digestion at $37^{\circ} \mathrm{C}$ overnight. Peptides were acidified with trifluoroacetic acid $(\sim 1 \%(\mathrm{v} / \mathrm{v})$ final concentration), desalted on homemade C18 StageTips and resuspended in $0.1 \%(\mathrm{v} / \mathrm{v})$ trifluoroacetic acid. Purified peptides were analysed by LC-MS/MS (see below).

MS data acquisition. Peptides were analysed by LC-MS/MS using an UltiMate 3000 RSLCnano system coupled online to a Q Exactive Plus Hybrid Quadrupole-Orbitrap mass spectrometer (both Thermo Fisher Scientific). Peptides were injected onto a C18-packed emitter in buffer A (2\% (v/v) acetonitrile, $0.5 \%(\mathrm{v} / \mathrm{v})$ acetic acid) and eluted with a linear 120-min gradient of 2\%-45\% (v/v) buffer B (80\% (v/v) acetonitrile, $0.5 \%$ $(\mathrm{v} / \mathrm{v})$ acetic acid). Eluting peptides were ionised in positive ion mode before data-dependent analysis. The target value for full scan MS spectra was $3 \times 10^{6}$ charges in the 300-1,650 $\mathrm{m} / \mathrm{z}$ range, with a resolution of 70,000. Ions were fragmented with normalised collision energy of 26, selecting the top 12 ions. A dynamic exclusion window of $30 \mathrm{~s}$ was enabled to avoid repeated sequencing of identical peptides. The target value for MS/MS spectra was $5 \times 10^{4}$ ions in the $200-2,000 \mathrm{~m} / z$ range, with a resolution of 17,500 . All spectra were acquired with 1 microscan and without lockmass. Two technical replicate injections were performed per sample for each of three independent biological experiments.

MS data analysis. Label-free quantitative analysis of MS data was performed using MaxQuant (version 1.5.3.17) ${ }^{81}$. Peptide lists were searched against the human UniProtKB database (version 2015_09) and a common contaminants database using the Andromeda search engine ${ }^{82}$. Cysteine carbamidomethylation was set as a fixed modification; methionine oxidation and protein $\mathrm{N}$-terminal acetylation were set as variable modifications (up to five modifications per peptide). MS data from two technical replicate analyses were merged for each corresponding independent biological experiment in the peptide search. Peptide and protein FDRs were set to 1\%, determined by applying a target-decoy search strategy using MaxQuant. Enzyme specificity was set as C-terminal to arginine and lysine, except when followed by proline, and a maximum of two missed cleavages were permitted in the database search. Minimum peptide length was seven amino acids, and at least one peptide ratio was required for label-free quantification. Proteins matching to the reversed or common contaminants databases were omitted.

Label-free quantification intensities for proteins quantified in at least two out of three biological replicate analyses of either Met1 or Met4 IACs were binary-logarithm-transformed. Data were normalised

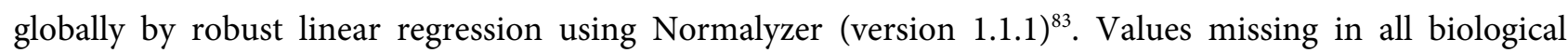
replicates of an experimental group were imputed using a first-pass single-value imputation, whereby the local minimum logarithm-transformed intensity value across a given replicate was used to impute missing-not-atrandom missing values ${ }^{84}$. Remaining missing values were imputed using a second-pass predictive mean matching imputation using the MICE $\mathrm{R}$ package (version 3.9.0 ${ }^{85}$. Statistical comparisons between experimental groups were carried out using two-sided Student's $t$-tests (for experimental groups with equal variance; F-test) or two-sided Welch's $t$-tests (where experimental groups displayed unequal variance; F-test) with Benjamini-Hochberg correction.

Functional enrichment analyses. Proteins were classified as actin-cytoskeletal proteins if they were annotated with Gene Ontology terms GO:0015629, GO:0030864, GO:0005884 or GO:0001725; actin-binding proteins were those annotated with terms GO:0003779 or GO:0051015; proteins involved in actin organisation were those annotated with terms GO:0030036, GO:0032956, GO:0030866, GO:0007015, GO:0110053, GO:0051639, GO:0043149 or GO:0051492; proteins involved in cell migration were those annotated with terms GO:0016477, GO:0030334, GO:0030335, GO:0001755, GO:0050900, GO:0043534, GO:0043536, GO:0043542, GO:0051451, GO:0010634, GO:0002042, GO:1901164, GO:0048870; proteins involved in ECM organisation, 
composition or binding were those annotated with terms GO:0030198, GO:0022617, GO:0090091, GO:1901201, GO:0050840, GO:0031012, GO:0005201, GO:0062023, GO:1904466; proteins involved in the immune response were those annotated with terms GO:0006955, GO:0045087, GO:0002250, GO:0002683, GO:0002768.

Over-representation analyses were performed using WebGestalt (version 2019) ${ }^{86}$. To reduce redundancy of enriched functional categories, where stated, gene sets were clustered according to Jaccard index and classified with representative terms using affinity propagation via the APCluster R package implemented in WebGestalt ${ }^{86,87}$.

Interaction network analysis. For analysis of the cSCC adhesome, a protein interaction network was constructed using curated physical protein-protein interactions from the BioGRID database as described previously ${ }^{88}$. To identify the functional active module, the maximal-scoring subnetwork was extracted from this interaction network using a diffusion-flow emulation model implemented in the BioNet $\mathrm{R}$ package (version 1.46.0) $)^{50}$. Briefly, $P$-values derived from statistical comparisons between experimental groups (twosided Student's or Welch's $t$-tests; see above) were converted into FDR-corrected scores based on signal-noise decomposition. Scores were assigned to corresponding nodes (proteins) to generate a weighted network (FDR $5 \%)^{88}$. The problem of finding a maximum-weight connected subnetwork was transformed into a prizecollecting Steiner tree (PCST) problem, and the exact solution to the latter was computed using integer linear programming implemented in the Heinz R package (version 1.63$)^{89}$.

For clustering the active module using the Louvain modularity maximisation method, edges were weighted by Spearman rank correlation coefficient +1 (ref. 88). We optimised the Girvan-Newman modularity quality function using the igraph R package (https://igraph.org). For clustering the active module using the constant Potts model, edges were weighted by Spearman rank correlation coefficient ${ }^{88}$. The problem of clustering was reformulated as a problem of finding the ground state of the Potts spin-glass model with a tuneable resolution parameter, $\gamma$ (ref. 90). We scanned $\gamma$ to maximise the Surprise quality function using the louvain Python package (https://pypi.org/project/louvain). Active modules were visualised using Cytoscape (version 3.6.1) $)^{91}$ via the CyREST API ${ }^{92}$.

For analysis of the core cSCC adhesion proteins, a composite functional association network was constructed using GeneMANIA (version 3.5.1; human interactions) ${ }^{93}$ in Cytoscape (version 3.8.0) ${ }^{91}$. The network was clustered using the edge-weighted force-directed algorithm in the Prefuse toolkit ${ }^{94}$.

Unsupervised learning. Binary, agglomerative hierarchical cluster analysis of $Z$-transformed protein or mRNA abundance was performed using Cluster 3.0 (C Clustering Library, version 1.54) ${ }^{95}$. Euclidean distance matrices were computed using average linkage, and clustering results were visualised using Java TreeView (version 1.1.5r2) ${ }^{96}$. For sample correlation analysis, Pearson correlation coefficient-based distance matrices were computed using complete linkage. Dimensionality reduction using principal component analysis was performed using R, and plots were annotated with 95\% confidence ellipses.

RNA isolation. RNA was extracted from Met4 and Met4 Mena $^{-/-}$cells using an RNeasy Mini kit (Qiagen) following the manufacturer's instructions. Homogenisation was performed by passing cells $(15 \times)$ through a 23 -gauge syringe. RNA was eluted from silica membranes in $30 \mu \mathrm{l}$ Rase-free water. An additional elution step was performed using the eluate of the first elution step to increase RNA yield. RNA sample concentrations were measured using a NanoDrop 2000c spectrophotometer (Thermo Fisher Scientific). All RNA samples had a ratio of absorbance at $260 \mathrm{~nm}$ and $280 \mathrm{~nm}$ (A260/280) of $\sim 2.0$. 
ENAH sequencing. The cloning and sequencing of ENAH from Met4 cells was performed as described previously $^{58}$. Briefly, $1 \mu \mathrm{g}$ of total RNA was used to synthesise cDNA using a SuperScript First-Strand kit (Invitrogen). cDNA was amplified using Phusion High-Fidelity DNA Polymerase (New England Biolabs) (forward primer, 5'-ATGAGTGAACAGAGTATCTG-3'; reverse primer, 5'-CTATGCAGTATTTGACTTGC$\left.3^{\prime}\right)$ in PCR reactions consisting of an initial denaturation step of $30 \mathrm{~s}$ at $95^{\circ} \mathrm{C}$, followed by 30 cycles at a denaturation temperature of $95^{\circ} \mathrm{C}\left(30 \mathrm{~s}\right.$ per cycle), an annealing temperature of $60^{\circ} \mathrm{C}(30 \mathrm{~s} \mathrm{per} \mathrm{cycle)}$ and an extension temperature of $72^{\circ} \mathrm{C}\left(1 \mathrm{~min}\right.$ per cycle). A final extension step was performed at $72^{\circ} \mathrm{C}(10 \mathrm{~min})$. PCR product was analysed on a $1 \%(\mathrm{w} / \mathrm{v})$ agarose gel, excised and purified using a gel extraction kit (Qiagen). PCR product was cloned into pCR-Blunt II-TOPO (Invitrogen) by blunt-end ligation according to manufacturer's instructions and transformed into DH5a cells. Plasmid was isolated using a QIAprep Miniprep kit (Qiagen) and sequenced using M13 sequencing primers (forward primer, 5'-TGTAAAACGACGGCCAGT-3'; reverse primer, 5'-CAGGAAACAGCTATGAC-3').

Multiplexed gene expression analysis. Total RNA was analysed using the 770-gene human nCounter PanCancer Progression panel (NanoString Technologies) according to manufacturer instructions. nCounter data were normalised to synthetic positive control targets and to housekeeping genes using nSolver Analysis software (version 4.0) (NanoString Technologies). A minimum mean count threshold was set to 20 counts, yielding a unimodal distribution of binary-logarithm-transformed counts. To remove between-batch variation, we used the pseudoreplicates approach of the removing unwanted variation-III normalisation method $^{97}$, applying $k=2$. Statistical comparisons between experimental groups were carried out using twosided Student's $t$-tests (for experimental groups with equal variance; F-test) or two-sided Welch's $t$-tests (where experimental groups displayed unequal variance; F-test) with Benjamini-Hochberg correction.

Lamin B1 DamID. Lentiviral transduction of Met4 and Met4 $\mathrm{Mena}^{-/-}$cells for lamin B1 DamID was performed as described previously ${ }^{98}$ with modifications. Briefly, HEK293T cells were co-transfected with psPAX2 (a lentiviral packaging plasmid), pMD2.G (which encodes vesicular stomatitis virus G protein) (both gifts from Val Brunton, University of Edinburgh) and a vector encoding either Dam fused to lamin B1 (pLgw Dam-lamin B1) or untethered Dam (pLgw Dam) (both gifts from Eric Schirmer, University of Edinburgh). Transfection medium was changed for fresh growth medium $18 \mathrm{~h}$ after transfection. Viral supernatant was harvested $72 \mathrm{~h}$ after transfection, filtered through a $0.45-\mu \mathrm{m}$ Millex-HA filter (Millipore), diluted in DMEM (2:1 DMEM:supernatant ratio), supplemented with polybrene and added to Met 4 and Met $4 \mathrm{Mena}^{-/-} \mathrm{cSCC}$ cells seeded on 6-well cell culture plates. cSCC cells were transferred to $15-\mathrm{cm}$ dishes $\left(1 \times 10^{6}\right.$ cells per dish $)$ and incubated for $60 \mathrm{~h}$, followed by genomic DNA (gDNA) extraction using a DNeasy Blood \& Tissue Kit (Qiagen) according to manufacturer instructions.

For methylated GATC library preparation, gDNA (300 ng) was digested using DpnI (New England Biolabs) for $6 \mathrm{~h}$ at $37^{\circ} \mathrm{C}$, followed by $D p n \mathrm{I}$ inactivation for $20 \mathrm{~min}$ at $80^{\circ} \mathrm{C}$. dsAdR adaptor ligation was performed using T4 DNA ligase (New England Biolabs) overnight at $16^{\circ} \mathrm{C}$, followed by ligase inactivation for $10 \mathrm{~min}$ at $65^{\circ} \mathrm{C}$. Digestion using DpnII (New England Biolabs) was then performed for $1 \mathrm{~h}$ at $37^{\circ} \mathrm{C}$, followed by $D p n I \mathrm{II}$ inactivation for $20 \mathrm{~min}$ at $65^{\circ} \mathrm{C}$. The reaction mixture was purified using a PCR purification kit (Qiagen) and eluted in RNase-free water. Initial PCR amplification using AdR_PCR primers and SYBR Select master mix was performed as described previously ${ }^{98}$ (PCR cycles 1-5), except the extension temperature was set at $72^{\circ} \mathrm{C}$. The optimum number of remaining cycles was determined by plotting linear $\mathrm{Rn}$ (passive reference dye-normalised reporter signal) versus cycle number and calculating the number of cycles corresponding to one-third of the height of the sigmoid curve to avoid PCR duplication. Methylated GATC gDNA fragments were purified using solid-phase reversible immobilisation beads and eluted in RNase-free water. GATC gDNA 
fragments (50 ng) were treated with Nextera $\mathrm{Tn} 5$ transposase (Illumina) for $5 \mathrm{~min}$ at $37^{\circ} \mathrm{C}$ with shaking. The transposase reaction was stopped by adding binding buffer from the PCR purification kit, and tagged DNA fragments were eluted in RNase-free water. DNA fragments were indexed and barcoded (Ad2.n) and PCRamplified using Ad1_noMX and Ad2.n primers ${ }^{99}$ and SYBR Select master mix, with initial holding temperature of $72^{\circ} \mathrm{C}$ ( $5 \mathrm{~min}$ ) followed by $98^{\circ} \mathrm{C}(30 \mathrm{~s})$. The optimum number of cycles was determined by qPCR (see below) at a denaturing temperature of $98^{\circ} \mathrm{C}(10 \mathrm{~s})$, annealing temperature of $63^{\circ} \mathrm{C}(30 \mathrm{~s})$ and extension temperature of $72^{\circ} \mathrm{C}(1 \mathrm{~min})$. Library fragments were purified using solid-phase reversible immobilisation beads and eluted in RNase-free water. Next-generation sequencing was performed using the NextSeq 500/550 high-output kit (version 2.5; 150 cycles) (Illumina, \#20024907) on the NextSeq 550 platform (Illumina, \#SY-415-1002).

Sequencing data analysis. Nextera adaptor trimming was performed using TrimGalore (version 0.6.3) (https://www.bioinformatics.babraham.ac.uk/projects/trim_galore) with the minimum required overlap with the adaptor sequence set to 5. Single-end read binning against the human reference genome (GRCh38) was performed using BBSplit, part of the BBMap package (version 38.11) (https://sourceforge.net/projects/bbmap). Binned reads were aligned (single-end mapping) to the human reference genome using Bowtie 2 (version 2.3.4.3) ${ }^{100}$. Mapped reads were sorted and indexed using SAMtools (version 1.6). The human reference genome was binned into GATC motifs fragments as described previously ${ }^{101}$ and expanded by $250 \mathrm{bp}$ on either side (500-bp fragment window). Single-end reads were imported as a GenomicRanges object in R, and the average number of reads within the 500-bp GATC window tilling the entire human chromosomes was computed. Read values were normalised to the total number of mapped reads (excluding decoy chromosomes). Ratios of Dam-lamin B1 versus untethered Dam control normalised reads were calculated and binary-logarithm-transformed, and data were visualised using the Integrated Genome Browser (version 9.1.4) ${ }^{102}$. Peak FDR analysis was performed using the find_peaks script (https://github.com/owenjm/find_peaks), with peak intensity threshold set to a minimum quantile of 75\%, stepping of 0.0005 and 15 iterations (peak FDR $<5 \%$ ).

qPCR analysis. RT-qPCR ( $1 \mu \mathrm{l}$ of cDNA) was performed on a StepOnePlus Real-Time PCR system using SYBR Select master mix (both Thermo Fisher Scientific). Reactions consisted of $10 \mathrm{~min}$ at $95^{\circ} \mathrm{C}$, followed by 40 cycles of $30 \mathrm{~s}$ at $95^{\circ} \mathrm{C}, 30 \mathrm{~s}$ at $60^{\circ} \mathrm{C}$ and $30 \mathrm{~s}$ at $72^{\circ} \mathrm{C}$. PTX3 gene expression (forward primer, $5^{\prime}$ CGTCTCTCCAGCAATGCATC-3'; reverse primer, 5'-AAGAGCTTGTCCCATTCCGA-3') was normalised to GAPDH (forward primer, 5'-GGACCTGACCTGCCGTCTAG-3'; reverse primer, 5'-TGGTGCTCAGTGTAGCCCAG-3'), calculating $\Delta \Delta$ Ct values.

For DamID-qPCR, methylated GATC gDNA fragments $(2.5 \mu \mathrm{l})$ were subjected to qPCR using SYBR Select master mix with qPCR reaction conditions as detailed above. Primers (TF2; forward primer, $5^{\prime}$ TACAAGGCGGGAAAATTGGC-3'; reverse primer, 5'-TATAACGGGCCAGTGGACTC-3') were used to amplify the putative PTX3 enhancer at genomic region 157,437,977-157,438,893 bp of chromosome 3 (human reference genome GRCh38). Statistical significance was assessed by two-sided Student's $t$-test.

Microarray data analysis. The expression of ENAH and PTX3 was assessed across a panel of patient-derived cSCC samples (GEO series accession identifier GSE98767) ${ }^{70}$. Data were binary-logarithm-transformed and normalised by cyclic loess using the limma R package (version 3.11) ${ }^{103}$. Scatter plot line-of-best-fit was generated using R, and linear correlation was assessed by Pearson correlation coefficient.

Statistics and reproducibility of experiments. Distributions of residuals were tested for normality using the Shapiro-Wilk test. Statistical significance of data with normally distributed residuals was calculated using a 
two-sided Student's or Welch's $t$-test (for comparing two unmatched groups) or an ordinary one-way ANOVA with Tukey's correction or Welch's one-way ANOVA with two-stage Benjamini-Krieger-Yekutieli correction (for comparing three unmatched groups). Statistical significance of data with distributions of residuals that departed from normality was calculated using a Kruskal-Wallis test with Dunn's correction or two-stage Benjamini-Krieger-Yekutieli correction (for comparing three unmatched groups). For proteomic data analysis, proteins quantified in at least two out of three independent biological replicates for at least one experimental condition were further analysed, and significantly differentially abundant proteins were determined using two-sided Student's $t$-tests (for experimental groups with equal variance; F-test) or twosided Welch's $t$-tests (for experimental groups with unequal variance; F-test) with Benjamini-Hochberg correction. Significantly differentially transcribed genes were determined using the same statistical tests as used for proteomic data analysis. For functional enrichment analyses, significantly enriched terms were determined using a hypergeometric test with Benjamini-Hochberg correction. No statistical method was used to predetermine sample size. Data plots were generated using Cytoscape, Excel (Microsoft), Integrated Genome Browser, Java TreeView, PlotsOfData ${ }^{104}$ or R.

\section{DATA AVAILABILITY}

Data supporting the findings of this study are available from the corresponding authors upon reasonable request. MS-based proteomics data have been deposited in ProteomeXchange (http://proteomecentral.proteomexchange.org) via the PRIDE partner repository with dataset accession identifier PXD021492.

\section{ACKNOWLEDGEMENTS}

We are grateful to Val Brunton, Francesca Di Modugno, Noor Gammoh, Frank Gertler and Eric Schirmer for reagents. We thank the Institute of Genetics and Cancer (IGC) Mass Spectrometry Facility for LC-MS/MS data acquisition; Alison Munro and the IGC Host and Tumour Profiling Unit Microarray Services for multiplexed gene expression analysis; Lizzie Freyer and the IGC Flow Cytometry Facility for flow cytometry analysis; Stephen Brown, Jeffrey Joseph and the IGC DNA Sequencing Facility for DNA sequencing; Amy Davies, Martin Lee, Laura Murphy, Matt Pearson and the IGC Advanced Imaging Resource for assistance with microscopy and image analysis; Richard Clark and the Edinburgh Clinical Research Facility for nextgeneration sequencing; Ainara Cabodevilla for assistance with lentiviral transduction; Laura Gómez-Cuadrado for assistance with RT-qPCR; Noor Gammoh for discussions and assistance with CRISPR/Cas9; Colin McLean for discussions and assistance with network analysis; Katerina Petelova for discussions and exploratory data analysis; Val Brunton, Gareth Inman, Roza Masalmeh, Bryan Serrels and Andy Sims for discussions. The work was funded by Cancer Research UK (grants C157/A15703 and C157/A24837 to M.C.F.). Development of the cSCC cell lines was funded by Cancer Research UK (grant A13044) and the European Research Council (grant 250170). The work made use of the resources provided by the Edinburgh Compute and Data Facility (ECDF), University of Edinburgh (https://www.ecdf.ed.ac.uk).

\section{AUTHOR CONTRIBUTIONS}

M.C.F. and A.B. conceived and co-ordinated the project; F.L.M.C., B.G.C.G., A.P.W., J.D.A., M.P., I.M.L., C.M.P., A.v.K., W.A.B., M.C.F. and A.B. designed the experiments and interpreted the results; F.L.M.C., B.B., A.L., Y.K. and J.C.W. performed the experiments; F.L.M.C., B.B. and A.B. analysed the data and prepared the figures; F.L.M.C., M.C.F. and A.B. wrote the paper; all authors commented on the manuscript and approved the final version. 


\section{COMPETING INTERESTS}

The authors declare no competing interests.

\section{REFERENCES}

1. Hamidi, H. \& Ivaska, J. Every step of the way: integrins in cancer progression and metastasis. Nat. Rev. Cancer 18, 533-548 https://doi.org/10.1038/s41568-018-0038-z (2018).

2. Kai, F., Drain, A. P. \& Weaver, V. M. The extracellular matrix modulates the metastatic journey. Dev. Cell 49, 332-346 https://doi.org/10.1016/j.devcel.2019.03.026 (2019).

3. Byron, A., Morgan, M. R. \& Humphries, M. J. Adhesion signalling complexes. Curr. Biol. 20, R1063R1067 https://doi.org/10.1016/j.cub.2010.10.059 (2010).

4. Green, H. J. \& Brown, N. H. Integrin intracellular machinery in action. Exp. Cell Res. 378, 226-231 https://doi.org/10.1016/j.yexcr.2019.03.011 (2019).

5. Geiger, B. \& Yamada, K. M. Molecular architecture and function of matrix adhesions. Cold Spring Harb. Perspect. Biol. 3, a005033 https://doi.org/10.1101/cshperspect.a005033 (2011).

6. Humphries, J. D., Chastney, M. R., Askari, J. A. \& Humphries, M. J. Signal transduction via integrin adhesion complexes. Curr. Opin. Cell Biol. 56, 14-21 https://doi.org/10.1016/j.ceb.2018.08.004 (2019).

7. Kechagia, J. Z., Ivaska, J. \& Roca-Cusachs, P. Integrins as biomechanical sensors of the microenvironment. Nat. Rev. Mol. Cell Biol. 20, 457-473 https://doi.org/10.1038/s41580-019-0134-2 (2019).

8. Zaidel-Bar, R., Itzkovitz, S., Ma'ayan, A., Iyengar, R. \& Geiger, B. Functional atlas of the integrin adhesome. Nat. Cell Biol. 9, 858-867 https://doi.org/10.1038/ncb0807-858 (2007).

9. Winograd-Katz, S. E., Fässler, R., Geiger, B. \& Legate, K. R. The integrin adhesome: from genes and proteins to human disease. Nat. Rev. Mol. Cell Biol. 15, 273-288 https://doi.org/10.1038/nrm3769 (2014).

10. Byron, A., Humphries, J. D., Bass, M. D., Knight, D. \& Humphries, M. J. Proteomic analysis of integrin adhesion complexes. Sci. Signal. 4, pt2 https://doi.org/10.1126/scisignal.2001827 (2011).

11. Kuo, J.-C., Han, X., Yates, J. R. III \& Waterman, C. M. Isolation of focal adhesion proteins for biochemical and proteomic analysis. Methods Mol. Biol. 757, 297-323 https://doi.org/10.1007/978-161779-166-6_19 (2012).

12. Jones, M. C. et al. Isolation of integrin-based adhesion complexes. Curr. Protoc. Cell Biol. 66, 9.8.19.8.15 https://doi.org/10.1002/0471143030.cb0908s66 (2015).

13. Robertson, J. et al. Characterization of the phospho-adhesome by mass spectrometry-based proteomics. Methods Mol. Biol. 1636, 235-251 https://doi.org/10.1007/978-1-4939-7154-1_15 (2017).

14. Byron, A. Proteomic profiling of integrin adhesion complex assembly. Methods Mol. Biol. 1764, 193236 https://doi.org/10.1007/978-1-4939-7759-8_13 (2018).

15. Humphries, J. D. et al. Proteomic analysis of integrin-associated complexes identifies RCC2 as a dual regulator of Rac1 and Arf6. Sci. Signal. 2, ra51 https://doi.org/10.1126/scisignal.2000396 (2009).

16. Kuo, J.-C., Han, X., Hsiao, C.-T., Yates, J. R. III \& Waterman, C. M. Analysis of the myosin-IIresponsive focal adhesion proteome reveals a role for $\beta$-Pix in negative regulation of focal adhesion maturation. Nat. Cell Biol. 13, 383-393 https://doi.org/10.1038/ncb2216 (2011).

17. Schiller, H. B., Friedel, C. C., Boulegue, C. \& Fässler, R. Quantitative proteomics of the integrin adhesome show a myosin II-dependent recruitment of LIM domain proteins. EMBO Rep. 12, 259-266 https://doi.org/10.1038/embor.2011.5 (2011). 
18. Byron, A., Humphries, J. D., Craig, S. E., Knight, D. \& Humphries, M. J. Proteomic analysis of $\alpha 4 \beta 1$ integrin adhesion complexes reveals $\alpha$-subunit-dependent protein recruitment. Proteomics 12, 21072114 https://doi.org/10.1002/pmic.201100487 (2012).

19. Schiller, H. B. et al. $\beta 1$ - and $\alpha$-class integrins cooperate to regulate myosin II during rigidity sensing of fibronectin-based microenvironments. Nat. Cell Biol. 15, 625-636 https://doi.org/10.1038/ncb2747 (2013).

20. Horton, E. R. et al. Definition of a consensus integrin adhesome and its dynamics during adhesion complex assembly and disassembly. Nat. Cell Biol. 17, 1577-1587 https://doi.org/10.1038/ncb3257 (2015).

21. Horton, E. R., Astudillo, P., Humphries, M. J. \& Humphries, J. D. Mechanosensitivity of integrin adhesion complexes: role of the consensus adhesome. Exp. Cell Res. 343, 7-13 https://doi.org/10.1016/j.yexcr.2015.10.025 (2016).

22. Kumeta, M., Yoshimura, S. H., Hejna, J. \& Takeyasu, K. Nucleocytoplasmic shuttling of cytoskeletal proteins: molecular mechanism and biological significance. Int. J. Cell Biol. 2012, 494902 https://doi.org/10.1155/2012/494902 (2012).

23. Wu, C. Migfilin and its binding partners: from cell biology to human diseases. J. Cell Sci. 118, 659-664 https://doi.org/10.1242/jcs.01639 (2005).

24. Zhou, J. L. et al. Zyxin promotes colon cancer tumorigenesis in a mitotic phosphorylation-dependent manner and through CDK8-mediated YAP activation. Proc. Natl Acad. Sci. USA 115, E6760-E6769 https://doi.org/10.1073/pnas.1800621115 (2018).

25. Hensley, P. J., Zetter, D., Horbinski, C. M., Strup, S. E. \& Kyprianou, N. Association of epithelialmesenchymal transition and nuclear cofilin with advanced urothelial cancer. Hum. Pathol. 57, 68-77 https://doi.org/10.1016/j.humpath.2016.06.020 (2016).

26. Jayo, A. et al. Fascin regulates nuclear movement and deformation in migrating cells. Dev. Cell 38, 371383 https://doi.org/10.1016/j.devcel.2016.07.021 (2016).

27. Thiam, H. R. et al. Perinuclear Arp2/3-driven actin polymerization enables nuclear deformation to facilitate cell migration through complex environments. Nat. Commun. 7, 10997 https://doi.org/10.1038/ncomms10997 (2016).

28. Desgrosellier, J. S. \& Cheresh, D. A. Integrins in cancer: biological implications and therapeutic opportunities. Nat. Rev. Cancer 10, 9-22 https://doi.org/10.1038/nrc2748 (2010).

29. Maziveyi, M. \& Alahari, S. K. Cell matrix adhesions in cancer: the proteins that form the glue. Oncotarget 8, 48471-48487 https://doi.org/10.18632/oncotarget.17265 (2017).

30. Kadrmas, J. L. \& Beckerle, M. C. The LIM domain: from the cytoskeleton to the nucleus. Nat. Rev. Mol. Cell Biol. 5, 920-931 https://doi.org/10.1038/nrm1499 (2004).

31. Byron, A. \& Frame, M. C. Adhesion protein networks reveal functions proximal and distal to cellmatrix contacts. Curr. Opin. Cell Biol. 39, 93-100 https://doi.org/10.1016/j.ceb.2016.02.013 (2016).

32. Hervy, M., Hoffman, L. \& Beckerle, M. C. From the membrane to the nucleus and back again: bifunctional focal adhesion proteins. Curr. Opin. Cell Biol. 18, 524-532 https://doi.org/10.1016/j.ceb.2006.08.006 (2006).

33. Wang, Y. \& Gilmore, T. D. Zyxin and paxillin proteins: focal adhesion plaque LIM domain proteins go nuclear. Biochim. Biophys. Acta 1593, 115-120 https://doi.org/10.1016/s0167-4889(02)00349-x (2003).

34. Kleinschmidt, E. G. \& Schlaepfer, D. D. Focal adhesion kinase signaling in unexpected places. Curr. Opin. Cell Biol. 45, 24-30 https://doi.org/10.1016/j.ceb.2017.01.003 (2017). 
35. Wang, N., Tytell, J. D. \& Ingber, D. E. Mechanotransduction at a distance: mechanically coupling the extracellular matrix with the nucleus. Nat. Rev. Mol. Cell Biol. 10, 75-82 https://doi.org/10.1038/nrm2594 (2009).

36. Kirby, T. J. \& Lammerding, J. Emerging views of the nucleus as a cellular mechanosensor. Nat. Cell Biol. 20, 373-381 https://doi.org/10.1038/s41556-018-0038-y (2018).

37. Wilhelmsen, K. et al. Nesprin-3, a novel outer nuclear membrane protein, associates with the cytoskeletal linker protein plectin. J. Cell Biol. 171, 799-810 https://doi.org/10.1083/jcb.200506083 (2005).

38. Kutscheidt, S. et al. FHOD1 interaction with nesprin-2G mediates TAN line formation and nuclear movement. Nat. Cell Biol. 16, 708-715 https://doi.org/10.1038/ncb2981 (2014).

39. Broders-Bondon, F., Ho-Bouldoires, T. H. N., Fernandez-Sanchez, M. E. \& Farge, E. Mechanotransduction in tumor progression: the dark side of the force. J. Cell Biol. 217, 1571-1587 https://doi.org/10.1083/jcb.201701039 (2018).

40. Guilluy, C. et al. Isolated nuclei adapt to force and reveal a mechanotransduction pathway in the nucleus. Nat. Cell Biol. 16, 376-381 https://doi.org/10.1038/ncb2927 (2014).

41. Izdebska, M., Zielinska, W., Grzanka, D. \& Gagat, M. The role of actin dynamics and actin-binding proteins expression in epithelial-to-mesenchymal transition and its association with cancer progression and evaluation of possible therapeutic targets. Biomed. Res. Int. 2018, 4578373 https://doi.org/10.1155/2018/4578373 (2018).

42. Pfisterer, K., Jayo, A. \& Parsons, M. Control of nuclear organization by F-actin binding proteins. Nucleus 8, 126-133 https://doi.org/10.1080/19491034.2016.1267093 (2017).

43. Hassan, S. et al. A unique panel of patient-derived cutaneous squamous cell carcinoma cell lines provides a preclinical pathway for therapeutic testing. Int. J. Mol. Sci. 20, 3428 https://doi.org/10.3390/ijms20143428 (2019).

44. Robertson, J. et al. Defining the phospho-adhesome through the phosphoproteomic analysis of integrin signalling. Nat. Commun. 6, 6265 https://doi.org/10.1038/ncomms7265 (2015).

45. Di Modugno, F. et al. The cytoskeleton regulatory protein hMena (ENAH) is overexpressed in human benign breast lesions with high risk of transformation and human epidermal growth factor receptor-2positive/hormonal receptor-negative tumors. Clin. Cancer Res. 12, 1470-1478 https://doi.org/10.1158/1078-0432.Ccr-05-2027 (2006).

46. Philippar, U. et al. A Mena invasion isoform potentiates EGF-induced carcinoma cell invasion and metastasis. Dev. Cell 15, 813-828 https://doi.org/10.1016/j.devcel.2008.09.003 (2008).

47. Pignatelli, J. et al. Invasive breast carcinoma cells from patients exhibit MenaINV-and macrophagedependent transendothelial migration. Sci. Signal. 7, ra112 https://doi.org/10.1126/scisignal.2005329 (2014).

48. Di Modugno, F. et al. Splicing program of human MENA produces a previously undescribed isoform associated with invasive, mesenchymal-like breast tumors. Proc. Natl Acad. Sci. USA 109, 19280-19285 https://doi.org/10.1073/pnas.1214394109 (2012).

49. Menche, J. et al. Uncovering disease-disease relationships through the incomplete interactome. Science 347, 1257601 https://doi.org/10.1126/science.1257601 (2015).

50. Beisser, D., Klau, G. W., Dandekar, T., Muller, T. \& Dittrich, M. T. BioNet: an R-package for the functional analysis of biological networks. Bioinformatics 26, 1129-1130 https://doi.org/10.1093/bioinformatics/btq089 (2010). 
51. Kassel, O. et al. A nuclear isoform of the focal adhesion LIM-domain protein Trip6 integrates activating and repressing signals at AP-1- and NF-kappaB-regulated promoters. Genes Dev. 18, 2518-2528 https://doi.org/10.1101/gad.322404 (2004).

52. Katz, Z. B. et al. $\beta$-Actin mRNA compartmentalization enhances focal adhesion stability and directs cell migration. Genes Dev. 26, 1885-1890 https://doi.org/10.1101/gad.190413.112 (2012).

53. Brackley, K. I. \& Grantham, J. Subunits of the chaperonin CCT interact with F-actin and influence cell shape and cytoskeletal assembly. Exp. Cell Res. 316, 543-553 https://doi.org/10.1016/j.yexcr.2009.11.003 (2010).

54. Zhang, Q. P. et al. Nesprin-2 is a multi-isomeric protein that binds lamin and emerin at the nuclear envelope and forms a subcellular network in skeletal muscle. J. Cell Sci. 118, 673-687 https://doi.org/10.1242/jcs.01642 (2005).

55. D'Alessandro, M. et al. Amphiphysin 2 orchestrates nucleus positioning and shape by linking the nuclear envelope to the actin and microtubule cytoskeleton. Dev. Cell 35, 186-198 https://doi.org/10.1016/j.devcel.2015.09.018 (2015).

56. Simpson, J. G. \& Roberts, R. G. Patterns of evolutionary conservation in the nesprin genes highlight probable functionally important protein domains and isoforms. Biochem. Soc. Trans. 36, 1359-1367 https://doi.org/10.1042/BST0361359 (2008).

57. Autore, F. et al. Large-scale modelling of the divergent spectrin repeats in nesprins: giant modular proteins. PLoS One 8, e63633 https://doi.org/10.1371/journal.pone.0063633 (2013).

58. Di Modugno, F. et al. Molecular cloning of hMena (ENAH) and its splice variant hMena+11a: epidermal growth factor increases their expression and stimulates hMena+11a phosphorylation in breast cancer cell lines. Cancer Res. 67, 2657-2665 https://doi.org/10.1158/0008-5472.CAN-06-1997 (2007).

59. Khatau, S. B. et al. A perinuclear actin cap regulates nuclear shape. Proc. Natl Acad. Sci. USA 106, 19017-19022 https://doi.org/10.1073/pnas.0908686106 (2009).

60. Thomas, D. G. et al. Non-muscle myosin IIB is critical for nuclear translocation during 3D invasion. J. Cell. Biol. 210, 583-594 https://doi.org/10.1083/jcb.201502039 (2015).

61. Kumar, A. \& Shivashankar, G. V. Dynamic interaction between actin and nesprin2 maintain the cell nucleus in a prestressed state. Methods Appl. Fluoresc. 4, 044008 https://doi.org/10.1088/20506120/4/4/044008 (2016).

62. Woroniuk, A. et al. STEF/TIAM2-mediated Rac1 activity at the nuclear envelope regulates the perinuclear actin cap. Nat. Commun. 9, 2124 https://doi.org/10.1038/s41467-018-04404-4 (2018).

63. Le, H. Q. et al. Mechanical regulation of transcription controls Polycomb-mediated gene silencing during lineage commitment. Nat. Cell Biol. 18, 864-875 https://doi.org/10.1038/ncb3387 (2016).

64. Tifft, K. E., Bradbury, K. A. \& Wilson, K. L. Tyrosine phosphorylation of nuclear-membrane protein emerin by Src, Abl and other kinases. J. Cell Sci. 122, 3780-3790 https://doi.org/10.1242/jcs.048397 (2009).

65. van Steensel, B. \& Belmont, A. S. Lamina-associated domains: links with chromosome architecture, heterochromatin, and gene repression. Cell 169, 780-791 https://doi.org/10.1016/j.cell.2017.04.022 (2017).

66. Oudin, M. J. et al. Tumor cell-driven extracellular matrix remodeling drives haptotaxis during metastatic progression. Cancer Discov. 6, 516-531 https://doi.org/10.1158/2159-8290.CD-15-1183 (2016).

67. Guelen, L. et al. Domain organization of human chromosomes revealed by mapping of nuclear lamina interactions. Nature 453, 948-951 https://doi.org/10.1038/nature06947 (2008). 
68. Bonavita, E. et al. PTX3 is an extrinsic oncosuppressor regulating complement-dependent inflammation in cancer. Cell 160, 700-714 https://doi.org/10.1016/j.cell.2015.01.004 (2015).

69. Robson, M. I. et al. Constrained release of lamina-associated enhancers and genes from the nuclear envelope during T-cell activation facilitates their association in chromosome compartments. Genome Res. 27, 1126-1138 https://doi.org/10.1101/gr.212308.116 (2017).

70. Inman, G. J. et al. The genomic landscape of cutaneous SCC reveals drivers and a novel azathioprine associated mutational signature. Nat. Commun. 9, 3667 https://doi.org/10.1038/s41467-018-06027-1 (2018).

71. Krause, M., Dent, E. W., Bear, J. E., Loureiro, J. J. \& Gertler, F. B. Ena/VASP proteins: regulators of the actin cytoskeleton and cell migration. Annu. Rev. Cell Dev. Biol. 19, 541-564 https://doi.org/10.1146/annurev.cellbio.19.050103.103356 (2003).

72. Uhler, C. \& Shivashankar, G. V. Regulation of genome organization and gene expression by nuclear mechanotransduction. Nat. Rev. Mol. Cell Biol. 18, 717-727 https://doi.org/10.1038/nrm.2017.101 (2017).

73. Barton, L. J., Soshnev, A. A. \& Geyer, P. K. Networking in the nucleus: a spotlight on LEM-domain proteins. Curr. Opin. Cell Biol. 34, 1-8 https://doi.org/10.1016/j.ceb.2015.03.005 (2015).

74. Demmerle, J., Koch, A. J. \& Holaska, J. M. The nuclear envelope protein emerin binds directly to histone deacetylase 3 (HDAC3) and activates HDAC3 activity. J. Biol. Chem. 287, 22080-22088 https://doi.org/10.1074/jbc.M111.325308 (2012).

75. Magrini, E., Mantovani, A. \& Garlanda, C. The dual complexity of PTX3 in health and disease: a balancing act? Trends Mol. Med. 22, 497-510 https://doi.org/10.1016/j.molmed.2016.04.007 (2016).

76. Serrels, B. et al. IL-33 and ST2 mediate FAK-dependent antitumor immune evasion through transcriptional networks. Sci. Signal. 10, eaan8355 https://doi.org/10.1126/scisignal.aan8355 (2017).

77. Serrels, A. et al. Nuclear FAK controls chemokine transcription, Tregs, and evasion of anti-tumor immunity. Cell 163, 160-173 https://doi.org/10.1016/j.cell.2015.09.001 (2015).

78. Proby, C. M. et al. Spontaneous keratinocyte cell lines representing early and advanced stages of malignant transformation of the epidermis. Exp. Dermatol. 9, 104-117 https://doi.org/10.1034/j.16000625.2000.009002104.x (2000).

79. Purdie, K. J., Pourreyron, C. \& South, A. P. Isolation and culture of squamous cell carcinoma lines. Methods Mol. Biol. 731, 151-159 https://doi.org/10.1007/978-1-61779-080-5_14 (2011).

80. Schindelin, J. et al. Fiji: an open-source platform for biological-image analysis. Nat. Methods 9, 676-682 https://doi.org/10.1038/nmeth.2019 (2012).

81. Cox, J. \& Mann, M. MaxQuant enables high peptide identification rates, individualized p.p.b.-range mass accuracies and proteome-wide protein quantification. Nat. Biotechnol. 26, 1367-1372 https://doi.org/10.1038/nbt.1511 (2008).

82. Cox, J. et al. Andromeda: a peptide search engine integrated into the MaxQuant environment. J. Proteome Res. 10, 1794-1805 https://doi.org/10.1021/pr101065j (2011).

83. Chawade, A., Alexandersson, E. \& Levander, F. Normalyzer: a tool for rapid evaluation of normalization methods for omics data sets. J. Proteome Res. 13, 3114-3120 https://doi.org/10.1021/pr401264n (2014).

84. Clough, T., Thaminy, S., Ragg, S., Aebersold, R. \& Vitek, O. Statistical protein quantification and significance analysis in label-free LC-MS experiments with complex designs. BMC Bioinformatics 13 Suppl 16, S6 https://doi.org/10.1186/1471-2105-13-S16-S6 (2012).

85. van Buuren, S. \& Groothuis-Oudshoorn, K. mice: multivariate imputation by chained equations in R. J. Stat. Softw. 45, 1-67 (2011). 
86. Liao, Y., Wang, J., Jaehnig, E. J., Shi, Z. \& Zhang, B. WebGestalt 2019: gene set analysis toolkit with revamped UIs and APIs. Nucleic Acids Res. 47, W199-W205 https://doi.org/10.1093/nar/gkz401 (2019).

87. Bodenhofer, U., Kothmeier, A. \& Hochreiter, S. APCluster: an R package for affinity propagation clustering. Bioinformatics 27, 2463-2464 https://doi.org/10.1093/bioinformatics/btr406 (2011).

88. Li Mow Chee, F. \& Byron, A. Network analysis of integrin adhesion complexes. Methods Mol. Biol. 2217 (in press).

89. Dittrich, M. T., Klau, G. W., Rosenwald, A., Dandekar, T. \& Müller, T. Identifying functional modules in protein-protein interaction networks: an integrated exact approach. Bioinformatics 24, i223-i231 https://doi.org/10.1093/bioinformatics/btn161 (2008).

90. Traag, V. A., Krings, G. \& Van Dooren, P. Significant scales in community structure. Sci. Rep. 3, 2930 https://doi.org/10.1038/srep02930 (2013).

91. Shannon, P. et al. Cytoscape: a software environment for integrated models of biomolecular interaction networks. Genome Res. 13, 2498-2504 https://doi.org/10.1101/gr.1239303 (2003).

92. Ono, K., Muetze, T., Kolishovski, G., Shannon, P. \& Demchak, B. CyREST: turbocharging Cytoscape access for external tools via a RESTful API. F1000Res 4, 478 https://doi.org/10.12688/f1000research.6767.1 (2015).

93. Montojo, J. et al. GeneMANIA Cytoscape plugin: fast gene function predictions on the desktop. Bioinformatics 26, 2927-2928 https://doi.org/10.1093/bioinformatics/btq562 (2010).

94. Heer, J., Card, S. K. \& Landay, J. A. Prefuse: a toolkit for interactive information visualization. In Proceedings of the SIGCHI Conference on Human Factors in Computing Systems 421-430 (ACM, New York, NY, USA, 2005).

95. de Hoon, M. J. L., Imoto, S., Nolan, J. \& Miyano, S. Open source clustering software. Bioinformatics 20, 1453-1454 https://doi.org/10.1093/bioinformatics/bth078 (2004).

96. Saldanha, A. J. Java Treeview-extensible visualization of microarray data. Bioinformatics 20, 32463248 https://doi.org/10.1093/bioinformatics/bth349 (2004).

97. Molania, R., Gagnon-Bartsch, J. A., Dobrovic, A. \& Speed, T. P. A new normalization for Nanostring nCounter gene expression data. Nucleic Acids Res. 47, 6073-6083 https://doi.org/10.1093/nar/gkz433 (2019).

98. Vogel, M. J., Peric-Hupkes, D. \& van Steensel, B. Detection of in vivo protein-DNA interactions using DamID in mammalian cells. Nat. Protoc. 2, 1467-1478 https://doi.org/10.1038/nprot.2007.148 (2007).

99. Buenrostro, J. D., Giresi, P. G., Zaba, L. C., Chang, H. Y. \& Greenleaf, W. J. Transposition of native chromatin for fast and sensitive epigenomic profiling of open chromatin, DNA-binding proteins and nucleosome position. Nat. Methods 10, 1213-1218 https://doi.org/10.1038/nmeth.2688 (2013).

100. Langmead, B. \& Salzberg, S. L. Fast gapped-read alignment with Bowtie 2. Nat. Methods 9, 357-359 https://doi.org/10.1038/nmeth.1923 (2012).

101. Marshall, O. J. \& Brand, A. H. damidseq_pipeline: an automated pipeline for processing DamID sequencing datasets. Bioinformatics 31, 3371-3373 https://doi.org/10.1093/bioinformatics/btv386 (2015)

102. Freese, N. H., Norris, D. C. \& Loraine, A. E. Integrated genome browser: visual analytics platform for genomics. Bioinformatics 32, 2089-2095 https://doi.org/10.1093/bioinformatics/btw069 (2016).

103. Ritchie, M. E. et al. limma powers differential expression analyses for RNA-sequencing and microarray studies. Nucleic Acids Res. 43, e47 https://doi.org/10.1093/nar/gkv007 (2015).

104. Postma, M. \& Goedhart, J. PlotsOfData-a web app for visualizing data together with their summaries. PLoS Biol. 17, e3000202 https://doi.org/10.1371/journal.pbio.3000202 (2019) 